\title{
Algorithmic Polynomials
}

\author{
Alexander A. Sherstov \\ University of California, Los Angeles \\ Los Angeles, California, USA \\ sherstov@cs.ucla.edu
}

\begin{abstract}
The approximate degree of a Boolean function $f\left(x_{1}, x_{2}, \ldots, x_{n}\right)$ is the minimum degree of a real polynomial that approximates $f$ pointwise within $1 / 3$. Upper bounds on approximate degree have a variety of applications in learning theory, differential privacy, and algorithm design in general. Nearly all known upper bounds on approximate degree arise in an existential manner from bounds on quantum query complexity.

We develop a first-principles, classical approach to the polynomial approximation of Boolean functions. We use it to give the first constructive upper bounds on the approximate degree of several fundamental problems:

(i) $O\left(n^{\frac{3}{4}-\frac{1}{4\left(2^{k}-1\right)}}\right)$ for the $k$-element distinctness problem;

(ii) $O\left(n^{1-\frac{1}{k+1}}\right)$ for the $k$-subset sum problem;

(iii) $O\left(n^{1-\frac{1}{k+1}}\right)$ for any $k$-DNF or $k$-CNF formula;

(iv) $O\left(n^{3 / 4}\right)$ for the surjectivity problem.
\end{abstract}

In all cases, we obtain explicit, closed-form approximating polynomials that are unrelated to the quantum arguments from previous work. Our first three results match the bounds from quantum query complexity. Our fourth result improves polynomially on the $\Theta(n)$ quantum query complexity of the problem and refutes the conjecture by several experts that surjectivity has approximate degree $\Omega(n)$. In particular, we exhibit the first natural problem with a polynomial gap between approximate degree and quantum query complexity.

\section{CCS CONCEPTS}

- Theory of computation $\rightarrow$ Algebraic complexity theory; Quantum complexity theory;

\section{KEYWORDS}

Approximate degree, quantum query complexity, $k$-element distinctness problem, $k$-subset sum problem, surjectivity problem, $k$-DNF formulas, $k$-CNF formulas

\section{ACM Reference Format:}

Alexander A. Sherstov. 2018. Algorithmic Polynomials. In Proceedings of 50th Annual ACM SIGACT Symposium on the Theory of Computing (STOC'18). ACM, New York, NY, USA, 14 pages. https://doi.org/10.1145/3188745.3188958

Permission to make digital or hard copies of all or part of this work for personal or classroom use is granted without fee provided that copies are not made or distributed for profit or commercial advantage and that copies bear this notice and the full citation on the first page. Copyrights for components of this work owned by others than ACM must be honored. Abstracting with credit is permitted. To copy otherwise, or republish, to post on servers or to redistribute to lists, requires prior specific permission and/or a fee. Request permissions from permissions@acm.org.

STOC'18, June 25-29, 2018, Los Angeles, CA, USA

(c) 2018 Association for Computing Machinery.

ACM ISBN 978-1-4503-5559-9/18/06 _\$15.00

https://doi.org/10.1145/3188745.3188958

\section{INTRODUCTION}

Let $f: X \rightarrow\{0,1\}$ be a given Boolean function, defined on a subset $X \subseteq\{0,1\}^{n}$. The $\epsilon$-approximate degree of $f$, denoted $\operatorname{deg}_{\epsilon}(f)$, is the minimum degree of a multivariate real polynomial $p$ such that $|f(x)-p(x)| \leqslant \epsilon$ for all $x \in X$. The standard setting of the error parameter for most applications is $\epsilon=1 / 3$, an aesthetically motivated constant that can be replaced by any other in $(0,1 / 2)$ at the expense of a constant-factor increase in approximate degree. The notion of approximate degree originated 25 years ago in the pioneering work of Nisan and Szegedy [22] and has since proved to be a powerful and versatile tool in theoretical computer science. Lower bounds on approximate degree have complexity-theoretic applications, whereas upper bounds are a tool in algorithm design. In the former category, the notion of approximate degree has enabled spectacular progress in circuit complexity, quantum query complexity, and communication complexity. On the algorithmic side, approximate degree underlies many of the strongest results obtained to date in computational learning, differentially private data release, and algorithm design in general. A detailed bibliographic overview of these complexity-theoretic and algorithmic applications is available in the full version [27] of our paper.

Despite these applications, progress in understanding approximate degree as a complexity measure has been slow and difficult. With very few exceptions [19, 22, 25, 26], all known upper bounds on approximate degree arise from quantum query algorithms. The connection between approximate degree and quantum query complexity was discovered by Beals et al. [7], who proved that the acceptance probability of an algorithm that makes $T$ queries is representable by a real polynomial of degree $2 T$. Put another way, every quantum algorithm implies an approximating polynomial of comparable complexity for the problem in question. Since the seminal work of Beals et al., essentially all upper bounds on approximate degree have come from quantum query algorithms, e.g., $[5,6,9,11,16-18,21,28]$. An illustrative example is the problem of determining the approximate degree of Boolean formulas of size $n$, posed in 2003 by O'Donnell and Servedio [23]. Progress on this question was stalled for a long time until it was finally resolved by Ambainis et al. [6], who built on the work of Farhi et al. [18] to give a near-optimal quantum query algorithm for any formula.

While quantum query complexity has been a fruitful source of approximate degree upper bounds, the exclusive reliance on quantum techniques for the polynomial approximation of Boolean functions is problematic. For one thing, a quantum query algorithm generally does not give any information about the approximating polynomial apart from its existence. For example, converting the quantum algorithms of $[5,6,9]$ to polynomials results in expressions so large and complicated that they are no longer meaningful. More importantly, quantum query algorithms are more constrained objects than real polynomials, and an optimal query algorithm for a given problem 
may be far less efficient than a polynomial constructed from scratch Given the many unresolved questions on approximate degree, there is a compelling need for polynomial approximation techniques that go beyond quantum query complexity.

In this paper, we take a fresh look at several breakthrough upper bounds for approximate degree, obtained over the years by sophisticated quantum query algorithms. In each case, we are able to construct an approximating polynomial from first principles that matches or improves on the complexity of the best quantum algorithm. All of our constructions produce explicit, closed-form polynomials that are unrelated to the corresponding quantum algorithms and are in the author's opinion substantially simpler. In one notable instance, our construction achieves a polynomial improvement on the complexity of the best possible quantum algorithm, refuting a conjecture [14] on the approximate degree of that problem and exhibiting the first natural example of a polynomial gap between approximate degree and quantum query complexity. Our proofs, discussed shortly, contribute novel techniques to the area.

\section{$1.1 k$-Element Distinctness}

The starting point in our work is the element distinctness problem $[2$, $3,5,9,12,20]$, which is one of the most studied questions in quantum query complexity and a major success story of the field. The input to the problem is a list of $n$ elements from a given range of size $r$, and the objective is to determine if the elements are pairwise distinct. A well-studied generalization of this problem is $k$-element distinctness, where $k$ is an arbitrary constant and the objective is to determine if some $k$-tuple of the elements are identical. Formally, the input to element distinctness and $k$-element distinctness is represented by a Boolean matrix $x \in\{0,1\}^{n \times r}$ in which every row $i$ has precisely one " 1 " entry, corresponding to the value of the $i$ th element. ${ }^{1}$ Aaronson and Shi [2], Ambainis [3], and Kutin [20] showed that element distinctness has quantum query complexity $\Omega\left(n^{2 / 3}\right)$. In follow-up work, Ambainis [5] gave a quantum algorithm for element distinctness with $O\left(n^{2 / 3}\right)$ queries, matching the lower bound in $[2,3,20]$. For the more general problem of $k$-element distinctness, Ambainis's algorithm [5] requires $O\left(n^{k /(k+1)}\right)$ queries. Using a different approach, Belovs [9] gave a polynomially faster algorithm for $k$-element distinctness, with query complexity

$$
O\left(n^{\frac{3}{4}-\frac{1}{4\left(2^{k}-1\right)}}\right) \equiv O\left(n^{1-\frac{1}{4\left(1-2^{-k}\right)}}\right) .
$$

Belovs's algorithm is currently the fastest known.

The algorithms of Ambainis [5] and Belovs [9] are highly nontrivial. The former is based on a quantum walk on the Johnson graph, whereas the latter uses the framework of learning graphs. We give an elementary, closed-form construction of an approximating polynomial for $k$-element distinctness that bypasses the quantum work. Formally, define $\mathrm{ED}_{n, r, k}:\{0,1\}_{\leqslant n}^{n \times r} \rightarrow\{0,1\}$ by

$$
\operatorname{ED}_{n, r, k}(x)= \begin{cases}1 & \text { if } x_{1, j}+x_{2, j}+\cdots+x_{n, j}<k \text { for each } j, \\ 0 & \text { otherwise }\end{cases}
$$

\footnotetext{
${ }^{1}$ Alternately, the input can be represented by a string of $n\lceil\log r\rceil$ bits. Switching to this more compact representation changes the complexity of the problem by a factor of at most $\lceil\log r\rceil$, which is negligible in all settings of interest.
}

The notation $\{0,1\}_{\leqslant n}^{n \times r}$ for the domain of this function indicates that we allow arbitrary input matrices $x \in\{0,1\}^{n \times r}$ of Hamming weight at most $n$, with no restriction on the placement of the " 1 " bits. This is of course a problem more general than $k$-element distinctness. We prove:

Theorem 1.1 ( $k$-element Distinctness). Let $k \geqslant 1$ be a fixed integer. Then for all $n, r \geqslant 1$,

$$
\operatorname{deg}_{1 / 3}\left(\mathrm{ED}_{n, r, k}\right)=O\left(\sqrt{n} \min \{n, r\}^{\frac{1}{2}-\frac{1}{4\left(1-2^{-k}\right)}}\right),
$$

where the approximating polynomial is given explicitly in each case.

Theorem 1.1 matches the quantum query bound (1) due to Belovs [9] and further generalizes it to every $r \geqslant 1$.

\section{$1.2 k$-Subset Sum, $k$-DNF and $k$-CNF Formulas}

Another well-studied problem in quantum query complexity is $k$ subset sum $[10,15]$. The input to this problem is a list of $n$ elements from a given finite Abelian group $G$, and the objective is to determine whether there is a $k$-tuple of elements that sum to 0 . Formally, the input is represented by a matrix $x \in\{0,1\}^{n \times|G|}$ with precisely one " 1 " entry in every row. Childs and Eisenberg [15] contributed an alternate analysis of Ambainis's algorithm for $k$-element distinctness [5] and showed how to adapt it to compute $k$-subset sum or any other function property with 1-certificate complexity at most $k$. In particular, any such problem has an approximating polynomial of degree $O\left(n^{k /(k+1)}\right)$. We give a first-principles construction of an approximating polynomial for any problem in this class, using techniques that are elementary and unrelated to the quantum work of Ambainis [5] and Childs and Eisenberg [15]. Our result is more general:

Theorem 1.2 ( $k$-DNF AND $k$-CNF Formulas). Let $k \geqslant 0$ be $a$ fixed integer. Let $f:\{0,1\}_{\leqslant n}^{N} \rightarrow\{0,1\}$ be representable on its domain by a $k$-DNF or $k-C N F$ formula. Then

$$
\operatorname{deg}_{1 / 3}(f)=O\left(n^{\frac{k}{k+1}}\right)
$$

where the approximating polynomial is given explicitly in each case.

Recall that a $k$-DNF formula in Boolean variables $x_{1}, x_{2}, \ldots, x_{N}$ is the disjunction of an arbitrary number of terms, where each term is the conjunction of at most $k$ literals from among $x_{1}, \overline{x_{1}}, \ldots, x_{N}, \overline{x_{N}}$. An essential aspect of Theorem 1.2 is that the approximate degree upper bound depends only on the Hamming weight $x_{1}+x_{2}+\cdots+x_{N}$ of the input and does not depend at all on the number of variables $N$, which can be arbitrarily large. Several special cases of Theorem 1.2 are worth noting. The theorem clearly applies to $k$-subset sum, which is by definition representable on its domain by a $k$-DNF formula. Moreover, in the terminology of Childs and Eisenberg [15], Theorem 1.2 applies to any function property with 1-certificate complexity at most $k$. Finally, taking $N=n$ shows that Theorem 1.2 applies to any function $f:\{0,1\}^{n} \rightarrow\{0,1\}$ representable by a $k$-DNF or $k$-CNF formula.

\subsection{Surjectivity}

While our proofs of Theorems 1.1 and 1.2 are significantly simpler than their quantum query counterparts, they do not give a 
quantitative improvement on previous work. This brings us to our next result. In the surjectivity problem [8], the input is a list of $n$ elements from a given range of size $r$, where $r \leqslant n$. The objective is to determine whether the input features all $r$ elements of the range. In function terminology, the input represents a mapping $\{1,2, \ldots, n\} \rightarrow\{1,2, \ldots, r\}$, and the objective is to determine whether the mapping is surjective. As usual in the quantum query literature, the input is represented by a Boolean matrix $x \in\{0,1\}^{n \times r}$ in which every row has precisely one " 1 " entry. Beame and Machmouchi [8] proved that for $r=\lfloor n / 2\rfloor+1$, the surjectivity problem has the maximum possible quantum query complexity, namely, $\Theta(n)$. This led several experts to conjecture that the approximate degree of surjectivity is also $\Theta(n)$; see, e.g., [14]. The conjecture was significant because its resolution would give the first $\mathrm{AC}^{0}$ circuit with approximate degree $\Theta(n)$, closing a long line of research [2, 3, 14, 22].

Surprisingly, we are able to show that surjectivity has an approximating polynomial of substantially lower degree, regardless of the range parameter $r$. Formally, let $\mathrm{SURJ}_{n, r}:\{0,1\}_{\leqslant n}^{n \times r} \rightarrow\{0,1\}$ be given by

$$
\operatorname{SURJ}_{n, r}(x)=\bigwedge_{j=1}^{r} \bigvee_{i=1}^{n} x_{i, j} .
$$

In keeping with our other results, our definition of $\mathrm{SURJ}_{n, r}$ allows arbitrary input matrices $\{0,1\}^{n \times r}$ of Hamming weight at most $n$. In this generalization of the surjectivity problem, the input can be thought of as an arbitrary relation rather than a function. We prove:

Theorem 1.3 (Surjectivity). For all positive integers $n$ and $r$,

$$
\operatorname{deg}_{1 / 3}\left(\operatorname{SURJ}_{n, r}\right)= \begin{cases}O\left(\sqrt{n} \cdot r^{1 / 4}\right) & \text { if } r \leqslant n, \\ 0 & \text { if } r>n,\end{cases}
$$

where the approximating polynomial is given explicitly in each case.

In particular, the theorem gives an approximating polynomial of degree $O\left(n^{3 / 4}\right)$ for all $r$. This upper bound is polynomially smaller than the problem's quantum query complexity $\Theta(n)$ for $r=\lfloor n / 2\rfloor+$ 1. While explicit functions with a polynomial gap between approximate degree and quantum query complexity have long been known [1, 4], Theorem 1.3 exhibits the first natural function with this property. The functions in previous work [1,4] were constructed with the specific purpose of separating complexity measures.

\subsection{Symmetric Functions}

Key building blocks in our proofs are symmetric Boolean functions $f:\{0,1\}^{n} \rightarrow\{0,1\}$. A classic result due to Paturi [24] states that the $1 / 3$-approximate degree of any such function $f$ is $\Theta(\sqrt{n \ell})$, where $\ell \in\{0,1,2, \ldots, n\}$ is the smallest number such that $f$ is constant on inputs of Hamming weight in $[\ell, n-\ell]$. When a symmetric function is used in an auxiliary role as part of a larger construction, it becomes important to have approximating polynomials for every possible setting of the error parameter, $1 / 2^{n} \leqslant \epsilon \leqslant 1 / 3$. A complete characterization of the $\epsilon$-approximate degree of symmetric functions for all $\epsilon$ was obtained by de Wolf [28], who sharpened previous bounds $[11,19,25]$ using an elegant quantum query algorithm. Prior to our work, no classical, first-principles proof was known for de Wolf's characterization, which is telling in view of the basic role that $\mathrm{AND}_{n}, \mathrm{OR}_{n}$, and other symmetric functions play in the area. We are able to give such a first-principles proof-in fact, three of them.

Theorem 1.4 (Symmetric Functions). Let $f:\{0,1\}^{n} \rightarrow\{0,1\}$ be a symmetric function. Let $\ell \in\{0,1,2, \ldots, n\}$ be an integer such that $f$ is constant on inputs of Hamming weight in $(\ell, n-\ell)$. Then for $1 / 2^{n} \leqslant \epsilon \leqslant 1 / 3$,

$$
\operatorname{deg}_{\epsilon}(f)=O\left(\sqrt{n \ell}+\sqrt{n \log \frac{1}{\epsilon}}\right),
$$

where the approximating polynomial is given explicitly in each case.

Theorem 1.4 matches de Wolf's quantum query result, tightly characterizing the $\epsilon$-approximate degree of every nonconstant symmetric function.

\subsection{Our Techniques}

Our proofs use only basic tools from approximation theory, such as Chebyshev polynomials. Our constructions additionally incorporate elements of classic algorithm design, e.g., the divide-andconquer paradigm, the inclusion-exclusion principle, and probabilistic reasoning. The title of our paper, "Algorithmic Polynomials," is a reference to this combination of classic algorithmic methodology and approximation theory. The informal message of our work is that algorithmic polynomials are not only more powerful than quantum algorithms but also easier to construct. A detailed discussion of Theorems 1.1-1.4 follows.

Extension theorem. As our starting point, we prove an extension theorem for polynomial approximation. This theorem allows one to construct an approximant for a given function $F$ using an approximant for a restriction $f$ of $F$. In more detail, consider an arbitrary function $f:\{0,1\}_{\leqslant m}^{N} \rightarrow[-1,1]$, defined on inputs $x \in\{0,1\}^{N}$ of Hamming weight at most $m$. Let $F_{n}:\{0,1\}_{\leqslant n}^{N} \rightarrow[-1,1]$ be the natural extension of $f$ to inputs of Hamming weight at most $n$, defined by $F_{n}=0$ outside the domain of $f$. From an approximationtheoretic point of view, a fundamental question to ask is how to efficiently "extend" any approximant for $f$ to an approximant for $F_{n}$. Unfortunately, this naïve formulation of the extension problem has no efficient solution; we describe a counterexample in Section 3. We are able to show, however, that the extension problem becomes meaningful if one works with $F_{2 m}$ instead of $f$. In other words, we give an efficient, explicit, black-box transformation of any approximant for the extension $F_{2 m}$ into an approximant for the extension $F_{n}$, for any $n \geqslant 2 m$. This result is essentially as satisfying as the "ideal" extension theorem in that the domains of $f$ and $F_{2 m}$ almost coincide and can be arbitrarily smaller than the domain of $F_{n}$. Our proof uses extrapolation bounds, extremal properties of Chebyshev polynomials, and ideas from rational approximation theory.

Symmetric functions. As mentioned earlier, we give three proofs of Theorem 1.4 on the $\epsilon$-approximate degree of symmetric functions. Each of the three proofs is fully constructive. Our simplest proof uses the extension theorem and is only half-a-page long. Here, we use brute-force interpolation to compute the function $f$ of interest on inputs of small Hamming weight, and then apply the extension 
theorem to effortlessly extend the interpolant to the full domain of $f$. Our second proof of Theorem 1.4 is an explicit, closed-form construction that uses Chebyshev polynomials as its only ingredient. This proof is a refinement of previous, suboptimal approximants for the AND function [19, 25]. We eliminate the inefficiency in previous work by using Chebyshev polynomials to achieve improved control at every point of the domain. Finally, our third proof of Theorem 1.4 is inspired by combinatorics rather than approximation theory. Here, we use a sampling experiment to construct an approximating polynomial for any symmetric function $f$ from an approximating polynomial for AND. In more detail, the experiment allows us to interpret $f$ as a linear combination of conjunctions of arbitrary degree, where the sum of the absolute values of the coefficients is reasonably small. Once such a representation is available, we simply replace every conjunction with its approximating polynomial. These substitutions increase the error of the approximation by a factor bounded by the sum of the absolute values of the coefficients in the original linear combination, which is negligible.

$k$-Element distinctness, $k-D N F$ and $k$-CNF formulas. We first establish an auxiliary result on the approximate degree of composed Boolean functions. Specifically, let $F: X \times\{0,1\}_{\leqslant n}^{N} \rightarrow\{0,1\}$ be given by $F(x, y)=\bigvee_{i=1}^{N} y_{i} \wedge f_{i}(x)$ for some set $X$ and some functions $f_{1}, f_{2}, \ldots, f_{N}: X \rightarrow\{0,1\}$. We bound the $\epsilon$-approximate degree of $F$ in terms of the approximate degree of $\bigvee_{i \in S} f_{i}$, maximized over all sets $S \subseteq\{1,2, \ldots, N\}$ of certain size. Crucially for our applications, the bound that we derive has no dependence on $N$. The proof uses Chebyshev polynomials and the inclusion-exclusion principle. Armed with this composition theorem, we give a short proof of Theorem 1.2 on the approximate degree of $k$-DNF and $k$-CNF formulas. The argument proceeds by induction on $k$, with the composition theorem invoked to implement the inductive step. The proof of Theorem 1.1 on the approximate degree of $k$-element distinctness is more subtle. It too proceeds by induction, with the composition theorem playing a central role. This time, however, the induction is with respect to both $k$ and the range parameter $r$, and the extension theorem is required to complete the inductive step. We note that we are able to bound the $\epsilon$-approximate degree of $k$-DNF formulas and $k$-element distinctness for every setting of $\epsilon$, rather than just $\epsilon=1 / 3$ in Theorems 1.1 and 1.2.

Surjectivity. Our proof of Theorem 1.3 is surprisingly short, given how improbable the statement was believed to be. As one can see from the defining equation for $\mathrm{SURJ}_{n, r}$, this function is the componentwise composition $\mathrm{AND}_{r} \circ \mathrm{OR}_{n}$ restricted to inputs of Hamming weight at most $n$. With this in mind, we start with a degree- $O(\sqrt{r})$ polynomial $\widehat{\mathrm{AND}}_{r}$ that approximates $\mathrm{AND}_{r}$ pointwise within $1 / 4$. The approximant in question is simply a scaled and shifted Chebyshev polynomial. It follows that the componentwise composition $\widehat{\mathrm{AND}}_{r} \circ \mathrm{OR}_{n}$, restricted to inputs of Hamming weight at most $n$, approximates $\mathrm{SURJ}_{n, r}$ pointwise within $1 / 4$. We are not finished, however, because the degree of $\widehat{\mathrm{AND}}_{r} \circ \mathrm{OR}_{n}$ is unacceptably large. Moving on, a few lines of algebra reveal that $\widehat{\mathrm{AND}}_{r} \circ \mathrm{OR}_{n}$ is a linear combination of conjunctions in which the absolute values of the coefficients sum to $2^{O(\sqrt{r})}$. It remains to approximate each of these conjunctions pointwise within $2^{-\Omega(\sqrt{r})}$ by a polynomial of degree
$O(\sqrt{n \sqrt{r}})=O\left(\sqrt{n} \cdot r^{1 / 4}\right)$, for which we use our explicit approximant from Theorem 1.4 along with the guarantee that the input has Hamming weight at most $n$. The proof of Theorem 1.3 is particularly emblematic of our work in its interplay of approximation-theoretic methodology (Chebyshev polynomials, linear combinations) and algorithmic thinking (reduction of the problem to the approximation of individual conjunctions).

We are pleased to report that our $O\left(n^{3 / 4}\right)$ upper bound for the surjectivity problem has just sparked further progress in the area by Bun, Kothari, and Thaler [13], who prove tight or nearly tight lower bounds on the approximate degree of several key problems in quantum query complexity. In particular, the authors of [13] prove that our upper bound for surjectivity is tight. We are confident that the ideas of our work will inform future research as well.

\section{PRELIMINARIES}

We start with a review of the technical preliminaries. The purpose of this section is to make the paper as self-contained as possible, and comfortably readable by a broad audience. The expert reader may wish to skim it for the notation or skip it altogether.

\subsection{Notation}

We view Boolean functions as mappings $X \rightarrow\{0,1\}$ for some finite set $X$. This arithmetization of the Boolean values "true" and "false" makes it possible to use Boolean operations in arithmetic expressions, as in $1-2 \bigvee_{i=1}^{n} x_{i}$. The familiar functions $\mathrm{OR}_{n}:\{0,1\}^{n} \rightarrow$ $\{0,1\}$ and $\mathrm{AND}_{n}:\{0,1\}^{n} \rightarrow\{0,1\}$ are given by $\mathrm{OR}_{n}(x)=\bigvee_{i=1}^{n} x_{i}$ and $\operatorname{AND}_{n}(x)=\bigwedge_{i=1}^{n} x_{i}=\prod_{i=1}^{n} x_{i}$. The negation of a Boolean function $f$ is denoted as usual by $\bar{f}=1-f$. The composition of $f$ and $g$ is denoted $f \circ g$, with $(f \circ g)(x)=f(g(x))$.

For a string $x \in\{0,1\}^{n}$, we denote its Hamming weight by $|x|=x_{1}+x_{2}+\cdots+x_{n}$. We use the following notation for strings of Hamming weight at most $k$, greater than $k$, and exactly $k$ :

$$
\begin{aligned}
\{0,1\}_{\leqslant k}^{n} & =\left\{x \in\{0,1\}^{n}:|x| \leqslant k\right\}, \\
\{0,1\}_{>k}^{n} & =\left\{x \in\{0,1\}^{n}:|x|>k\right\}, \\
\{0,1\}_{k}^{n} & =\left\{x \in\{0,1\}^{n}:|x|=k\right\} .
\end{aligned}
$$

For a string $x \in\{0,1\}^{n}$ and a set $S \subseteq\{1,2, \ldots, n\}$, we let $\left.x\right|_{S}$ denote the restriction of $x$ to the indices in $S$. In other words, $\left.x\right|_{S}=$ $x_{i_{1}} x_{i_{2}} \ldots x_{i_{|S|} \mid}$, where $i_{1}<i_{2}<\cdots<i_{|S|}$ are the elements of $S$. The characteristic vector of a subset $S \subseteq\{1,2, \ldots, n\}$ is denoted $\mathbf{1}_{S}$.

We let $\mathbb{N}=\{0,1,2,3, \ldots\}$ and $[n]=\{1,2, \ldots, n\}$. For a set $S$ and a real number $k$, we define

$$
\left(\begin{array}{l}
S \\
k
\end{array}\right)=\{A \subseteq S:|A|=k\}, \quad\left(\begin{array}{c}
S \\
\leqslant k
\end{array}\right)=\{A \subseteq S:|A| \leqslant k\} .
$$

We analogously define $\left(\begin{array}{c}S \\ \geqslant k\end{array}\right),\left(\begin{array}{c}S \\ <k\end{array}\right)$, and $\left(\begin{array}{c}S \\ >k\end{array}\right)$. We let $\ln x$ and $\log x$ stand for the natural logarithm of $x$ and the logarithm of $x$ to base 2 , respectively. For a logical condition $C$, we use the Iverson bracket notation

$$
\mathbf{I}[C]= \begin{cases}1 & \text { if } C \text { holds } \\ 0 & \text { otherwise }\end{cases}
$$

For a function $f: X \rightarrow \mathbb{R}$ on a finite set $X$, we use the standard norms $\|f\|_{\infty}=\max _{x \in X}|f(x)|$ and $\|f\|_{1}=\sum_{x \in X}|f(x)|$. 
Let $S_{n}$ denote the symmetric group on $n$ elements. For a permutation $\sigma \in S_{n}$ and a string $x=\left(x_{1}, x_{2}, \ldots, x_{n}\right)$, we adopt the shorthand $\sigma x=\left(x_{\sigma(1)}, x_{\sigma(2)}, \ldots, x_{\sigma(n)}\right)$. A function $f\left(x_{1}, x_{2}, \ldots, x_{n}\right)$ is called symmetric if it is invariant under permutations of the input variables: $f\left(x_{1}, x_{2}, \ldots, x_{n}\right) \equiv f\left(x_{\sigma(1)}, x_{\sigma(2)}, \ldots, x_{\sigma(n)}\right)$ for all $x$ and $\sigma$.

\subsection{Approximate Degree}

Recall that the total degree of a multivariate real polynomial $p: \mathbb{R}^{n} \rightarrow$ $\mathbb{R}$, denoted $\operatorname{deg} p$, is the largest degree of any monomial of $p$. We use the terms "degree" and "total degree" interchangeably in this paper. This paper studies the approximate representation of functions of interest by polynomials. Specifically, let $f: X \rightarrow \mathbb{R}$ be a given function, for a finite subset $X \subset \mathbb{R}^{n}$. Define

$$
E(f, d)=\min _{p: \operatorname{deg} p \leqslant d}\|f-p\|_{\infty},
$$

where the minimum is over polynomials of degree at most $d$. In words, $E(f, d)$ is the least error to which $f$ can be approximated by a real polynomial of degree at most $d$. For a real number $\epsilon \geqslant 0$, the $\epsilon$-approximate degree of $f$ is defined as

$$
\operatorname{deg}_{\epsilon}(f)=\min \{d: E(f, d) \leqslant \epsilon\} .
$$

Thus, $\operatorname{deg}_{\epsilon}(f)$ is the least degree of a real polynomial that approximates $f$ pointwise to within $\epsilon$. We refer to any such polynomial as a uniform approximant for $f$ with error $\epsilon$. In the study of Boolean functions $f$, the standard setting of the error parameter is $\epsilon=1 / 3$. This constant is chosen mostly for aesthetic reasons and can be replaced by any other constant in $(0,1 / 2)$ at the expense of a constant-factor increase in approximate degree. The following fact on the exact representation of functions by polynomials is well known; a short proof is included in the full version [27] of this paper.

FACт 2.1. For every function $f:\{0,1\}^{N} \leqslant n \rightarrow \mathbb{R}$,

$$
\operatorname{deg}_{0}(f) \leqslant n \text {. }
$$

\subsection{Chebyshev Polynomials}

Recall from Euler's identity that

$$
(\cos x+\mathbf{i} \sin x)^{d}=\cos d x+\mathbf{i} \sin d x, \quad d=0,1,2, \ldots,
$$

where $\mathbf{i}$ denotes the imaginary unit. Multiplying out the left-hand side and using $\sin ^{2} x=1-\cos ^{2} x$, we obtain a univariate polynomial $T_{d}$ of degree $d$ such that

$$
T_{d}(\cos x)=\cos d x .
$$

This unique polynomial is the Chebyshev polynomial of degree $d$. The representation (3) immediately reveals all the roots of $T_{d}$, and all the extrema of $T_{d}$ in the interval $[-1,1]$ :

$$
\begin{array}{ll}
T_{d}\left(\cos \left(\frac{2 i-1}{2 d} \pi\right)\right)=0, & i=1,2, \ldots, d, \\
T_{d}\left(\cos \left(\frac{i}{d} \pi\right)\right)=(-1)^{i}, & i=0,1, \ldots, d, \\
\left|T_{d}(t)\right| \leqslant 1, & t \in[-1,1] .
\end{array}
$$

The extremum at 1 is of particular significance, and we note it separately:

$$
T_{d}(1)=1
$$

In view of (2), the defining equation (3) implies that

$$
\begin{aligned}
T_{d}(\cos x) & =\sum_{i=0}^{\lfloor d / 2\rfloor}\left(\begin{array}{l}
d \\
2 i
\end{array}\right)(-1)^{i}(\sin x)^{2 i}(\cos x)^{d-2 i} \\
& =\sum_{i=0}^{\lfloor d / 2\rfloor}\left(\begin{array}{l}
d \\
2 i
\end{array}\right)\left(\cos ^{2} x-1\right)^{i}(\cos x)^{d-2 i},
\end{aligned}
$$

so the leading coefficient of $T_{d}$ is $\sum_{i=0}^{\lfloor d / 2\rfloor}\left(\begin{array}{c}d \\ 2 i\end{array}\right)=2^{d-1}$ for $d \geqslant 1$. As a result, we have the factored representation

$$
T_{d}(t)=2^{d-1} \prod_{i=1}^{d}\left(t-\cos \left(\frac{2 i-1}{2 d} \pi\right)\right), \quad d \geqslant 1 .
$$

By (2) and (3),

$$
\begin{aligned}
T_{d}(\cos x) & =\cos d x \\
& =\frac{1}{2}(\cos x-\mathbf{i} \sin x)^{d}+\frac{1}{2}(\cos x+\mathbf{i} \sin x)^{d} \\
& =\frac{1}{2}\left(\cos x-\mathrm{i} \sqrt{1-\cos ^{2} x}\right)^{d}+\frac{1}{2}\left(\cos x+\mathrm{i} \sqrt{1-\cos ^{2} x}\right)^{d},
\end{aligned}
$$

whence

$$
T_{d}(t)=\frac{1}{2}\left(t-\sqrt{t^{2}-1}\right)^{d}+\frac{1}{2}\left(t+\sqrt{t^{2}-1}\right)^{d}, \quad|t| \geqslant 1 .
$$

The following fundamental fact follows from (9) by elementary calculus.

Fact 2.2 (Derivative of Chebyshev polynomials). For any integer $d \geqslant 0$ and real $t \geqslant 1$,

$$
T_{d}^{\prime}(t) \geqslant d^{2}
$$

Together, (9) and Fact 2.2 give the following useful lower bound for Chebyshev polynomials on $[1, \infty)$.

Proposition 2.3. For any integer $d \geqslant 1$,

$$
\begin{array}{ll}
T_{d}(1+\delta) \geqslant 1+d^{2} \delta, & 0 \leqslant \delta<\infty, \\
T_{d}(1+\delta) \geqslant 2^{d \sqrt{\delta}-1} & 0 \leqslant \delta \leqslant 1 .
\end{array}
$$

Proof. The first bound follows from the intermediate value theorem in view of (7) and Fact 2.2. For the second bound, use (9) to write

$$
T_{d}(1+\delta) \geqslant \frac{1}{2}\left(1+\delta+\sqrt{(1+\delta)^{2}-1}\right)^{d} \geqslant \frac{1}{2}(1+\sqrt{\delta})^{d} \geqslant \frac{2^{d \sqrt{\delta}}}{2},
$$

where the last step uses $1+x \geqslant 2^{x}$ for $x \in[0,1]$.

\subsection{The Conjunction Norm}

Recall that a conjunction in Boolean variables $x_{1}, \ldots, x_{n}$ is the AND of some subset of the literals $x_{1}, \overline{x_{1}}, \ldots, x_{n}, \overline{x_{n}}$. Analogously, a disjunction is the OR of some subset of $x_{1}, \overline{x_{1}}, \ldots, x_{n}, \overline{x_{n}}$. We regard conjunctions and disjunctions as Boolean functions $\{0,1\}^{n} \rightarrow$ $\{0,1\}$ and in particular as a special case of real functions $\{0,1\}^{n} \rightarrow$ $\mathbb{R}$. For a subset $X \subseteq\{0,1\}^{n}$ and a function $f: X \rightarrow \mathbb{R}$, we define the conjunction norm $\Pi(f)$ to be the minimum $\Lambda \geqslant 0$ such that

$$
f(x)=\lambda_{1} C_{1}(x)+\lambda_{2} C_{2}(x)+\cdots+\lambda_{N} C_{N}(x) \quad(x \in X)
$$

for some integer $N$, some conjunctions $C_{1}, C_{2}, \ldots, C_{N}$, and some real coefficients $\lambda_{1}, \lambda_{2}, \ldots, \lambda_{N}$ with $\left|\lambda_{1}\right|+\left|\lambda_{2}\right|+\cdots+\left|\lambda_{N}\right| \leqslant \Lambda$. Our choice of the symbol $\Pi$, for "product," is motivated by the view 
of conjunctions as products of literals. The next proposition, proved in the full version [27] of this paper, shows that $\Pi$ is a norm on the space of multivariate real functions and establishes other useful properties of this complexity measure.

Proposition 2.4 (Conjunction Norm). Let $f, g: X \rightarrow \mathbb{R}$ be given functions, for a nonempty set $X \subseteq\{0,1\}^{n}$. Then:

(i) $\Pi(f) \geqslant 0$, with equality if and only if $f=0$;

(ii) $\Pi(\lambda f)=|\lambda| \Pi(f)$ for any real $\lambda$;

(iii) $\Pi(f+g) \leqslant \Pi(f)+\Pi(g)$;

(iv) $\Pi(f \cdot g) \leqslant \Pi(f) \Pi(g)$;

(v) $\Pi(f) \leqslant\|f\|_{1}$;

(vi) $\Pi(f) \leqslant 2$ if $f$ is a disjunction.

\section{THE EXTENSION THEOREM}

This section establishes an approximation-theoretic result of independent interest, the extension theorem, that we use several times in the rest of the paper to construct approximating polynomials. To set the stage for this result, let $f:\{0,1\}_{\leqslant m}^{N} \rightarrow[-1,1]$ be a given function, defined on inputs of Hamming weight up to $m$. For any integer $n>m$, consider the extension $F_{n}$ of $f$ to inputs of Hamming weight up to $n$, given by

$$
F_{n}(x)= \begin{cases}f(x) & \text { if }|x| \leqslant m, \\ 0 & \text { otherwise }\end{cases}
$$

From the point of view of approximation theory, a fundamental question to ask is how to "extend" any approximant for $f$ to an approximant for $F_{n}$, without degrading the quality of the approximation or significantly increasing the approximant's degree. Ideally, we would like the approximant for the extension $F_{n}$ to have degree within a small factor of the original degree, e.g., a factor of $O(n / m)^{\alpha}$ for some constant $0<\alpha<1$.

Unfortunately, the extension problem is hopeless as stated. Indeed, consider the special case of the constant function $f=1$, so that

$$
F_{n}(x)= \begin{cases}1 & \text { if } 0 \leqslant|x| \leqslant m, \\ 0 & \text { if } m<|x| \leqslant n .\end{cases}
$$

In this example, $\operatorname{deg}_{1 / 3}(f)=0$ but $\operatorname{deg}_{1 / 3}\left(F_{n}\right)=\Omega(\sqrt{n})$ by a wellknown result of Nisan and Szegedy [22]. In particular, there is no efficient way to transform an approximant for a general function $f$ into an approximant for the extension $F_{n}$. Our contribution is to show that the extension problem becomes meaningful and efficiently solvable if one's starting point is an approximant for $F_{2 m}$ rather than for $f$. In other words, we give an efficient, black-box transformation of an approximant for $F_{2 m}$ into an approximant for any extension $F_{n}$, where $n \geqslant 2 m$. The formal statement of our result is as follows.

Theorem 3.1 (Extension theorem). Let $f:\{0,1\}_{\leqslant m}^{N} \rightarrow[-1,1]$ be given, where $N \geqslant m \geqslant 0$ are integers. For integers $n \geqslant m$, define $F_{n}:\{0,1\}_{\leqslant n}^{N} \rightarrow[-1,1]$ by

$$
F_{n}(x)= \begin{cases}f(x) & \text { if }|x| \leqslant m \\ 0 & \text { otherwise. }\end{cases}
$$

Then for some absolute constant $C>1$ and all $\epsilon, \delta \in(0,1 / 2)$ and $n \geqslant m$,

$$
\operatorname{deg}_{\epsilon+\delta}\left(F_{n}\right) \leqslant C \sqrt{\frac{n}{m+1}} \cdot\left(\operatorname{deg}_{\epsilon}\left(F_{2 m}\right)+\log \frac{1}{\delta}\right) .
$$

Theorem 3.1 solves the extension problem with only a factor- $\sqrt{n / m}$ increase in degree. The approximation quality of the new approximant can be made arbitrarily close to that of the original at a small additive cost in degree. This overhead in degree and error is optimal, as we will discover in applications later in this paper. We also note that the constant 2 in this result was chosen exclusively for aesthetic reasons, and (10) holds with $F_{2 m}$ replaced by $F_{\lceil\mathrm{cm}\rceil}$ for any constant $c>1$. The proof of Theorem 3.1 is available in the full version [27] of this paper.

\section{SYMMETRIC FUNCTIONS}

In this section, we study the approximation of symmetric functions. This class includes $\mathrm{AND}_{n}$ and $\mathrm{OR}_{n}$, which are fundamental building blocks of our constructions in the rest of the paper. Our result here is as follows.

THeorem 4.1. Let $f:\{0,1\}^{n} \rightarrow[-1,1]$ be an arbitrary symmetric function. Let $k$ be a nonnegative integer such that $f$ is constant on inputs of Hamming weight in $(k, n-k)$. Then for $0<\epsilon<1 / 2$,

$$
\operatorname{deg}_{\epsilon}(f)=O\left(\sqrt{n k}+\sqrt{n \log \frac{1}{\epsilon}}\right),
$$

where the approximating polynomial is given explicitly in each case.

Theorem 4.1 is tight [25] for every $\epsilon \in\left[1 / 2^{n}, 1 / 3\right]$ and every symmetric function $f:\{0,1\}^{n} \rightarrow\{0,1\}$, with the obvious exception of the constant functions $f=0$ and $f=1$. Prior to our work, de Wolf [28] proved the upper bound (11) by giving an $\epsilon$-error quantum query algorithm for any symmetric function $f$. The novelty of Theorem 4.1 is the construction of an explicit, closed-form approximating polynomial that achieves de Wolf's upper bound. We give three proofs of Theorem 4.1, corresponding to Sections 4.1-4.3 below.

\subsection{A Proof Using the Extension Theorem}

Our first proof of Theorem 4.1 is based on the extension theorem, and is the shortest of the three. The centerpiece of the proof is the following technical lemma, in which we construct a closed-form approximant for any function supported on inputs of low Hamming weight.

Lemma 4.2. Let $f:\{0,1\}^{n} \rightarrow[-1,1]$ be given. Let $k$ be a nonnegative integer such that $f(x)=0$ for $|x|>k$. Then for $0<\epsilon<1 / 2$,

$$
\operatorname{deg}_{\epsilon}(f)=O\left(\sqrt{n k}+\sqrt{n \log \frac{1}{\epsilon}}\right),
$$

where the approximating polynomial is given explicitly in each case.

Proof. Abbreviate $m=\left\lceil k+\log \frac{1}{\epsilon}\right\rceil$. If $m \geqslant n$, the bound in the theorem statement follows trivially from $\operatorname{deg}_{0}(f) \leqslant n$. In the rest of the proof, we focus on the complementary case $m<n$. 
For $i \geqslant m$, define $F_{i}:\{0,1\}_{\leqslant i}^{n} \rightarrow[-1,1]$ by

$$
F_{i}(x)= \begin{cases}f(x) & \text { if }|x| \leqslant m \\ 0 & \text { otherwise }\end{cases}
$$

Then

$$
\begin{aligned}
\operatorname{deg}_{\epsilon}(f) & =\operatorname{deg}_{\epsilon}\left(F_{n}\right) \\
& \leqslant \sqrt{\frac{n}{m}} \cdot O\left(\operatorname{deg}_{0}\left(F_{2 m}\right)+\log \frac{1}{\epsilon}\right) \\
& \leqslant \sqrt{\frac{n}{m}} \cdot O\left(2 m+\log \frac{1}{\epsilon}\right) \\
& =O\left(\sqrt{n k}+\sqrt{n \log \frac{1}{\epsilon}}\right),
\end{aligned}
$$

where the first step uses $f=F_{n}$, the second step applies the extension theorem (Theorem 3.1), and the third step is valid by Fact 2.1. Moreover, the approximating polynomial is given explicitly because the extension theorem and Fact 2.1 are fully constructive.

We are now in a position to prove the claimed result on the approximation of arbitrary symmetric functions.

Theorem 4.3. Let $f:\{0,1\}^{n} \rightarrow[-1,1]$ be given. Let $k$ be a nonnegative integer such that $f$ is constant on inputs of Hamming weight in $(k, n-k)$. Then for $0<\epsilon<1 / 2$,

$$
\operatorname{deg}_{\epsilon}(f)=O\left(\sqrt{n k}+\sqrt{n \log \frac{1}{\epsilon}}\right),
$$

where the approximating polynomial is given explicitly in each case.

A powerful feature of Theorem 4.3 is that the function of interest is only assumed to be symmetric on inputs of Hamming weight in $(k, n-k)$. In particular, Theorem 4.3 is significantly more general than Theorem 4.1 .

Proof of Theorem 4.3. If $k \geqslant n / 2$, the theorem follows from the trivial bound $\operatorname{deg}_{0}(f) \leqslant n$. For the complementary case $k<n / 2$, write

$$
f\left(x_{1}, \ldots, x_{n}\right)=\lambda+f^{\prime}\left(x_{1}, \ldots, x_{n}\right)+f^{\prime \prime}\left(\overline{x_{1}}, \ldots, \overline{x_{n}}\right),
$$

where $\lambda \in[-1,1]$ and $f^{\prime}, f^{\prime \prime}:\{0,1\}^{n} \rightarrow[-2,2]$ are functions that vanish on $\{0,1\}_{>k}^{n}$. Then

$$
\begin{aligned}
\operatorname{deg}_{\epsilon}(f) & \leqslant \max \left\{\operatorname{deg}_{\epsilon / 2}\left(f^{\prime}\right), \operatorname{deg}_{\epsilon / 2}\left(f^{\prime \prime}\right)\right\} \\
& \leqslant \max \left\{\operatorname{deg}_{\epsilon / 4}\left(\frac{f^{\prime}}{2}\right), \operatorname{deg}_{\epsilon / 4}\left(\frac{f^{\prime \prime}}{2}\right)\right\} \\
& =O\left(\sqrt{n k}+\sqrt{n \log \frac{1}{\epsilon}}\right),
\end{aligned}
$$

where the last step uses Lemma 4.2.

\subsection{A Proof from First Principles}

We now present our second proof of Theorem 4.1. This proof proceeds from first principles, using Chebyshev polynomials as its only ingredient. To convey the construction as clearly as possible, we first present an approximant for the simplest and most important symmetric function, $\mathrm{AND}_{n}$. For this, we adopt the strategy of previous constructions [19, 25], whereby one first zeroes out as many of the integer points $n-1, n-2, n-3, \ldots$ as possible and then uses a Chebyshev polynomial to approximate $\mathrm{AND}_{n}$ on the remaining points of $\{0,1,2, \ldots, n\}$. We depart from the previous work in the implementation of the first step. Specifically, we produce the zeroes using a product of Chebyshev polynomials, each of which is stretched and shifted so as to obtain an extremum at $n$ and a root at one of the points $n-1, n-2, n-3, \ldots$ The use of Chebyshev polynomials allows us to avoid explosive growth at the nonzeroes, thereby eliminating a key source of inefficiency in $[19,25]$. The lemma below shows how to produce a single zero, at any given point $m$.

Lemma 4.4. Let $n$ and $m$ be given integers, $0 \leqslant m<n$. Then there is a univariate polynomial $T_{n, m}$ such that

$$
\begin{aligned}
& T_{n, m}(n)=1, \\
& T_{n, m}(m)=0 \\
& \left|T_{n, m}(t)\right| \leqslant 1 \\
& \operatorname{deg}\left(T_{n, m}\right) \leqslant\left\lceil\frac{\pi}{4} \sqrt{\frac{n}{n-m}}\right\rceil .
\end{aligned}
$$

Proof. As mentioned above, the construction involves starting with a Chebyshev polynomial and stretching and shifting it so as to move an extremum to $n$ and a root to $m$. In more detail, let

$$
d=\left\lceil\frac{\pi}{4} \sqrt{\frac{n}{n-m}}\right\rceil .
$$

Consider the linear map $L$ that sends

$$
\begin{aligned}
& L(n)=1, \\
& L(m)=\cos \left(\frac{\pi}{2 d}\right) .
\end{aligned}
$$

Observe that under $L$, the length of any given interval of the real line changes by a factor of

$$
\frac{1}{n-m}\left(1-\cos \frac{\pi}{2 d}\right) \leqslant \frac{1}{n-m}\left(1-\left(1-\frac{\pi^{2}}{8 d^{2}}\right)\right)=\frac{\pi^{2}}{8 d^{2}(n-m)} \leqslant \frac{2}{n},
$$

where the first step uses $\cos x \geqslant 1-\frac{1}{2} x^{2}$ for $x \in \mathbb{R}$. In particular,

$$
\begin{aligned}
L([0, n]) & \subseteq\left[L(n)-\frac{2}{n} \cdot n, L(n)\right] \\
& \subseteq[-1,1] .
\end{aligned}
$$

We now show that the sought properties (12)-(15) hold for the polynomial $T_{n, m}(t)=T_{d}(L(t))$, where $T_{d}$ denotes as usual the Chebyshev polynomial of degree $d$. To start with, $T_{n, m}(n)=$ $T_{d}(L(n))=T_{d}(1)=1$, where the last two steps use (16) and (7), respectively. Similarly, $T_{n, m}(m)=T_{d}(L(m))=T_{d}(\cos (\pi /(2 d)))=$ $\cos (\pi / 2)=0$, where the second and third steps follow from (17) and (3), respectively. Continuing, $T_{n, m}([0, n])=T_{d}(L([0, n])) \subseteq$ $T_{d}([-1,1]) \subseteq[-1,1]$, where the last two steps follow from (18) and (6), respectively. Finally, the degree bound (15) is immediate from the choice of $d$.

We now obtain the desired approximant for AND and OR, using the two-stage approach described earlier. 
THEOREM 4.5. For some constant $c>0$ and all integers $n \geqslant 1$ and $d \geqslant 0$, there is an (explicitly given) univariate polynomial $p$ such that

$$
\begin{array}{ll}
p(n)=1, & \\
|p(t)| \leqslant \exp \left(-\frac{c d^{2}}{n}\right), & t=0,1,2, \ldots, n-1, \\
|p(t)| \leqslant 1, & t \in[0, n], \\
\operatorname{deg} p \leqslant d . &
\end{array}
$$

In particular,

$$
\begin{array}{ll}
E\left(\mathrm{AND}_{n}, d\right) \leqslant \frac{1}{2} \exp \left(-\frac{c}{2} \cdot \frac{d^{2}}{n}\right), & d=0,1,2,3, \ldots, \\
\operatorname{deg}_{\epsilon}\left(\mathrm{AND}_{n}\right) \leqslant O\left(\sqrt{n \log \frac{1}{\epsilon}}\right), & 0<\epsilon<\frac{1}{2},
\end{array}
$$

and analogously

$$
\begin{array}{ll}
E\left(\mathrm{OR}_{n}, d\right) \leqslant \frac{1}{2} \exp \left(-\frac{c}{2} \cdot \frac{d^{2}}{n}\right), & d=0,1,2,3, \ldots \\
\operatorname{deg}_{\epsilon}\left(\mathrm{OR}_{n}\right) \leqslant O\left(\sqrt{n \log \frac{1}{\epsilon}}\right), & 0<\epsilon<\frac{1}{2} .
\end{array}
$$

Proof. For $d \geqslant n$, we may simply take $p(t)=t(t-1)(t-$ 2) $\cdots(t-n+1) / n$ !. In what follows, we focus on the construction of $p$ for $d<n$. Let $\ell, r$ be integer parameters to be chosen later, where $1 \leqslant \ell \leqslant n-1$ and $1 \leqslant r \leqslant n$. We define

$$
p(t)=\frac{T_{r}(t /(n-\ell))}{T_{r}(n /(n-\ell))} \prod_{i=n-\ell+1}^{n-1} T_{n, i}(t),
$$

where $T_{n, i}$ is as constructed in Lemma 4.4 , and $T_{r}$ stands as usual for the Chebyshev polynomial of degree $r$. By (12) and (13),

$$
\begin{aligned}
& p(n)=1, \\
& p(t)=0, \quad t=n-\ell+1, \ldots, n-1 .
\end{aligned}
$$

Moreover,

$$
\begin{aligned}
\max _{0 \leqslant t \leqslant n-\ell}|p(t)| & =\max _{0 \leqslant t \leqslant n-\ell} \frac{\left|T_{r}(t /(n-\ell))\right|}{\left|T_{r}(n /(n-\ell))\right|} \prod_{i=n-\ell+1}^{n-1}\left|T_{n, i}(t)\right| \\
& \leqslant \frac{1}{\left|T_{r}(n /(n-\ell))\right|} \\
& \leqslant \frac{1}{\max \left\{1+\frac{r^{2} \ell}{n}, 2^{r \sqrt{\ell / n}-1}\right\}} \\
& \leqslant \frac{1}{\min \left\{\exp \left(\frac{r^{2} \ell}{3 n}\right), \exp \left(\frac{r \sqrt{\ell}}{3 \sqrt{n}}\right)\right\}}
\end{aligned}
$$

where the second step uses (6) and (14); the third step follows from Proposition 2.3; and the last step uses $1+x \geqslant \exp (x / 3)$ for $0 \leqslant x \leqslant 4$, and $2^{\sqrt{x}-1} \geqslant \exp (\sqrt{x} / 3)$ for $x \geqslant 4$. Next,

$$
\begin{aligned}
\max _{0 \leqslant t \leqslant n}|p(t)| & =\max _{0 \leqslant t \leqslant n} \frac{\left|T_{r}(t /(n-\ell))\right|}{\left|T_{r}(n /(n-\ell))\right|} \prod_{i=n-\ell+1}^{n-1}\left|T_{n, i}(t)\right| \\
& \leqslant \max _{0 \leqslant t \leqslant n} \frac{\left|T_{r}(t /(n-\ell))\right|}{\left|T_{r}(n /(n-\ell))\right|} \\
& \leqslant 1,
\end{aligned}
$$

where the second inequality uses (14), and the third inequality follows from (6), (7), and Fact 2.2. Finally,

$$
\begin{aligned}
\operatorname{deg} p & \leqslant r+\sum_{i=n-\ell+1}^{n-1} \operatorname{deg}\left(T_{n, i}\right) \\
& \leqslant r+\sum_{i=1}^{\ell-1}\left(\frac{\pi}{4} \sqrt{\frac{n}{i}}+1\right) \\
& \leqslant r+\ell-1+\frac{\pi \sqrt{n}}{4} \int_{0}^{\ell-1} \frac{d t}{\sqrt{t}} \\
& =r+\ell-1+\frac{\pi \sqrt{n(\ell-1)}}{2} \\
& \leqslant r+3 \sqrt{n(\ell-1)},
\end{aligned}
$$

where the second step uses (15). Now (19)-(22) follow from (27)-(31) by setting $r=\lceil d / 2\rceil$ and $\ell=\left\lfloor d^{2} /(36 n)\right\rfloor+1$.

The remaining claims in the theorem statement follow in a straightforward manner from (19)-(22). For (23), we have

$$
\begin{aligned}
E\left(\mathrm{AND}_{n}, d\right) & \leqslant \max _{x \in\{0,1\}^{n}}\left|\operatorname{AND}_{n}(x)-\frac{p\left(\sum_{i=1}^{n} x_{i}\right)}{1+\exp \left(-c d^{2} / n\right)}\right| \\
& \leqslant \frac{\exp \left(-c d^{2} / n\right)}{1+\exp \left(-c d^{2} / n\right)} \\
& \leqslant \frac{1}{2} \exp \left(-\frac{c}{2} \cdot \frac{d^{2}}{n}\right)
\end{aligned}
$$

where the last step uses $a /(1+a) \leqslant \sqrt{a} / 2$ for any $a \geqslant 0$. This in turn settles (25) since $\mathrm{OR}_{n}(x)=1-\mathrm{AND}_{n}\left(1-x_{1}, \ldots, 1-x_{n}\right)$. Finally, (24) and (26) are immediate from (23) and (25), respectively.

Theorem 4.5 readily generalizes to arbitrary symmetric functions, thereby giving a new proof of Theorem 4.1. Complete details are available in the full version [27] of this paper.

\subsection{A Proof Using a Sampling Argument}

In the full version [27] of this paper, we give a third proof of Theorem 4.1, inspired by combinatorics rather than approximation theory. There, we show how to approximate an arbitrary symmetric function $f$ using an approximant for AND (cf. Theorem 4.5) and a sampling argument. Suppose for the sake of concreteness that $f$ is supported on inputs of Hamming weight at most $k$. Given a string $x \in\{0,1\}^{n}$, consider the experiment whereby one chooses $\lfloor n / k\rfloor$ bits of $x$ independently and uniformly at random, and outputs the disjunction of those bits. To approximate $f$, we feed the expected value of the sampling experiment to a suitable univariate polynomial constructed by Lagrange interpolation. The expected value of the experiment as a function of $x$ has $\Pi$-norm at most 2, which by Proposition 2.4 means that the overall composition has small $\Pi$-norm as well. To finish the proof, we expand the composition as a linear combination of conjunctions and replace each conjunction by a corresponding approximant from Theorem 4.5 .

\subsection{Generalizations}

Theorem 4.5 on the approximation of AND and OR obviously generalizes to arbitrary conjunctions and disjunctions. Somewhat less obviously, it generalizes in an optimal manner to conjunctions and disjunctions whose domain of definition is restricted to the first 
few levels of the hypercube. We record this generalization for later use; see the full version [27] of this paper for a detailed proof.

Theorem 4.6. Let $f:\{0,1\}_{\leqslant n}^{N} \rightarrow\{0,1\}$ be given by

$$
f(x)=\left(\bigvee_{i \in A} x_{i}\right) \vee\left(\bigvee_{i \in B} \overline{x_{i}}\right)
$$

for some subsets $A, B \subseteq\{1,2, \ldots, N\}$. Then

$$
E(f, d) \leqslant \frac{1}{2} \exp \left(-\frac{c d^{2}}{n}\right), \quad d=0,1,2, \ldots,
$$

where $c>0$ is an absolute constant. Moreover, the approximating polynomial is given explicitly in each case.

Corollary 4.7. Let $f:\{0,1\}_{\leqslant n}^{N} \rightarrow\{0,1\}$ be given $b y$

$$
f(x)=\left(\bigwedge_{i \in A} x_{i}\right) \wedge\left(\bigwedge_{i \in B} \overline{x_{i}}\right)
$$

for some subsets $A, B \subseteq\{1,2, \ldots, N\}$. Then

$$
E(f, d) \leqslant \frac{1}{2} \exp \left(-\frac{c d^{2}}{n}\right), \quad d=0,1,2, \ldots,
$$

where $c>0$ is an absolute constant. Moreover, the approximating polynomial is given explicitly in each case.

Proof. Apply Theorem 4.6 to $1-f$.

\section{$5 \quad k$-DNF AND $k$-CNF FORMULAS}

Recall that a $k-D N F$ formula in Boolean variables $x_{1}, x_{2}, \ldots, x_{N}$ is the disjunction of zero or more terms, where each term is the conjunction of at most $k$ literals from among $x_{1}, \overline{x_{1}}, x_{2}, \overline{x_{2}}, \ldots, x_{N}, \overline{x_{N}}$. As a convention, we consider the constant functions 0 and 1 to be valid $k$-DNF formulas for every $k \geqslant 0$. Analogously, a $k-C N F$ formula in Boolean variables $x_{1}, x_{2}, \ldots, x_{N}$ is the conjunction of zero or more clauses, where each clause is the disjunction of at most $k$ literals from among $x_{1}, \overline{x_{1}}, x_{2}, \overline{x_{2}}, \ldots, x_{N}, \overline{x_{N}}$. Again, we consider the constant functions 0 and 1 to be valid $k$-CNF formulas for all $k \geqslant 0$. Recall that a function $f$ is representable by a $k$-DNF formula if and only if its negation $\bar{f}$ is representable by a $k$-CNF formula. Note also that the definition of $k$-DNF formulas is hereditary in the sense that a $k$-DNF formula is also a $k^{\prime}$-DNF formula for any $k^{\prime} \geqslant k$, and analogously for CNF formulas.

The contribution of this section is to settle Theorem 1.2 on the approximate degree of every $k$-DNF and $k$-CNF formula. We will in fact prove the following more precise result, for every setting of the error parameter.

Theorem 5.1. Let $f:\{0,1\}_{\leqslant n}^{N} \rightarrow\{0,1\}$ be representable on its domain by a $k-D N F$ or $k-C N F$ formula. Then

$$
\operatorname{deg}_{\epsilon}(f) \leqslant c \cdot(\sqrt{2})^{k} n \frac{k}{k+1}\left(\log \frac{1}{\epsilon}\right)^{\frac{1}{k+1}}
$$

for all $0<\epsilon<1 / 2$, where $c>1$ is an absolute constant independent of $f, N, n, k, \epsilon$. Moreover, the approximating polynomial is given explicitly in each case.

We present the proof of this theorem in Sections 5.1-5.4 below.

\subsection{Key Quantities}

For nonnegative integers $n$ and $k$ and a real number $\Delta \geqslant 1$, we define $D(n, k, \Delta)=\max _{f} \operatorname{deg}_{2^{-}}(f)$, where the maximum is over all functions $f:\{0,1\}^{N} \leqslant n \rightarrow\{0,1\}$ for some $N \geqslant n$ that are representable by a $k$-DNF formula. Fact 2.1 gives the upper bound

$$
D(n, k, \Delta) \leqslant n \text {. }
$$

Since the only 0 -DNF formulas are the constant functions 0 and 1 , we obtain

$$
D(n, 0, \Delta)=0 .
$$

We will prove Theorem 5.1 by induction of $k$, with (34) serving as the base case.

\subsection{A Composition Theorem}

The inductive step in our analysis of $D(n, k, \Delta)$ relies on a certain general bound on approximate degree for a class of composed functions, as follows. Its proof is available in the full version [27] of our paper.

Theorem 5.2. Let $F: X \times\{0,1\}_{\leqslant n}^{N} \rightarrow\{0,1\}$ be given by

$$
F(x, y)=\bigvee_{i=1}^{N} y_{i} \wedge f_{i}(x)
$$

for some functions $f_{1}, f_{2}, \ldots, f_{N}: X \rightarrow\{0,1\}$. Then

$$
\begin{aligned}
& \operatorname{deg}_{\epsilon}(F) \leqslant C \sqrt{n b \log \frac{1}{\epsilon}} \\
&+ \max _{S \subseteq\{1, \ldots, N\}} \operatorname{deg} \exp \left(-C \sqrt{\frac{n}{b} \log \frac{1}{\epsilon}}\right)\left(\bigvee_{i \in S} f_{i}\right) \\
&|S| \leqslant C \sqrt{n b \log \frac{1}{\epsilon}}
\end{aligned}
$$

for all reals $b \geqslant 1$ and $0<\epsilon \leqslant 1 / 2$, where $C>1$ is an absolute constant independent of $F, N, n, b, \epsilon$.

\subsection{A Recursive Bound}

Using Theorem 5.2 as our main tool, we now derive the promised recurrence for $D(n, k, \Delta)$.

LEMMA 5.3. There is a constant $C \geqslant 1$ such that for all integers $n, k \geqslant 1$ and reals $\Delta \geqslant 1$,

$$
D(n, k, \Delta) \leqslant \max _{b \geqslant 1}\left\{C \sqrt{n b \Delta}+D\left(n, k-1, \Delta+C \sqrt{\frac{n \Delta}{b}}\right)\right\} .
$$

Proof. Let $f:\{0,1\}_{\leqslant n}^{N} \rightarrow\{0,1\}$ be a $k$-DNF formula. Our objective is to bound $\operatorname{deg}_{2^{-\Delta}}(f)$ by the right-hand side of (36). We may assume that

$$
f \not \equiv 1 \text {, }
$$

since the bound holds trivially for the constant function $f=1$.

Write $f=f^{\prime} \vee f^{\prime \prime}$, where $f^{\prime}$ is a $k$-DNF formula in which every term has an unnegated variable, and $f^{\prime \prime}$ is a $k$-DNF formula whose terms feature only negated variables. Collecting like terms in $f^{\prime}$, we immediately obtain the following for some $(k-1)$-DNF formulas $f_{1}^{\prime}, f_{2}^{\prime}, \ldots, f_{N}^{\prime}$ :

$$
f^{\prime}(x)=\bigvee_{i=1}^{N} x_{i} \wedge f_{i}^{\prime}(x)
$$


We now turn to $f^{\prime \prime}$. By (37), there exists $x^{*} \in\{0,1\}_{\leqslant n}^{N}$ such that $f^{\prime \prime}\left(x^{*}\right)=0$. Consider the subset $I=\left\{i: x_{i}^{*}=1\right\}$, of cardinality

$$
|I| \leqslant n
$$

Since every occurrence of a variable in $f^{\prime \prime}(x)$ is negated, we conclude that every term in $f^{\prime \prime}(x)$ features some literal $\overline{x_{i}}$ with $i \in I$. Collecting like terms, we obtain the representation

$$
f^{\prime \prime}(x)=\bigvee_{i \in I} \overline{x_{i}} \wedge f_{i}^{\prime \prime}(x)
$$

where each $f_{i}^{\prime \prime}$ is a $(k-1)$-DNF formula.

To summarize (38)-(40), the function $f=f^{\prime} \vee f^{\prime \prime}$ is a subfunction of some $F:\{0,1\}_{\leqslant n}^{N} \times\{0,1\}_{\leqslant 2 n}^{N+n} \rightarrow\{0,1\}$ of the form

$$
F(x, y)=\bigvee_{i=1}^{N+n} y_{i} \wedge f_{i}(x)
$$

where each $f_{i}$ is a $(k-1)$-DNF formula. Now

$$
\begin{aligned}
& \operatorname{deg}_{2^{-}}(f) \\
& \leqslant \operatorname{deg}_{2^{-}-}(F) \\
& \leqslant \max _{b \geqslant 1}\left\{c \sqrt{2 n b \Delta}+\max _{S \subseteq\{1,2 \ldots, N+n\}} \operatorname{deg}_{2^{-\Delta}} \exp (-c \sqrt{2 n \Delta / b})\left(\bigvee_{i \in S} f_{i}\right)\right\} \\
& \leqslant \max _{b \geqslant 1}\left\{c \sqrt{2 n b \Delta}+D\left(n, k-1, \Delta+\frac{c}{\ln 2} \sqrt{\frac{2 n \Delta}{b}}\right)\right\},
\end{aligned}
$$

where the second step follows from Theorem 5.2 for a suitable absolute constant $c \geqslant 1$, and the third step is justified by the fact that each $\bigvee_{i \in S} f_{i}:\{0,1\}_{\leqslant n}^{N} \rightarrow\{0,1\}$ is a $(k-1)$-DNF formula. In conclusion, (36) holds with $C=c \sqrt{2} / \ln 2$.

\subsection{Solving the Recurrence}

It remains to solve the recurrence for $D(n, k, \Delta)$ given by (34) and Lemma 5.3.

THEOREM 5.4. There is a constant $c \geqslant 1$ such that for all integers $n, k \geqslant 0$ and reals $\Delta \geqslant 1$,

$$
D(n, k, \Delta) \leqslant c \cdot(\sqrt{2})^{k} n^{\frac{k}{k+1}} \Delta^{\frac{1}{k+1}} .
$$

This result settles Theorem 5.1. Indeed, if $f:\{0,1\}_{\leqslant n}^{N} \rightarrow\{0,1\}$ is representable by a $k$-DNF formula, then (32) is immediate from (41). The same bound applies to $k$-CNF formulas because they are negations of $k$-DNF formulas, and $\operatorname{deg}_{\epsilon}(f)=\operatorname{deg}_{\epsilon}(1-f)$ for any $f$.

Proof of Theorem 5.4. We will prove (41) for $c=2(C+1)^{2}$, where $C \geqslant 1$ is the absolute constant from Lemma 5.3. The proof is by induction on $k$. The base $k=0$ is valid due to (34). For the inductive step, let $k \geqslant 1$ be arbitrary. For $\Delta \geqslant n$, the claim is immediate from (33), and we focus on the complementary case

$$
1 \leqslant \Delta \leqslant n
$$

For every $b \geqslant 1$,

$D(n, k, \Delta)$

$$
\begin{aligned}
& \leqslant \min \left\{n, C \sqrt{n b \Delta}+D\left(n, k-1, \Delta+C \sqrt{\frac{n \Delta}{b}}\right)\right\} \\
& \leqslant \min \left\{n, C \sqrt{n b \Delta}+2(C+1)^{2} 2^{\frac{k-1}{2}} n^{\frac{k-1}{k}}\left(\Delta+C \sqrt{\frac{n \Delta}{b}}\right)^{\frac{1}{k}}\right\} \\
& \leqslant(C+1) \sqrt{n b \Delta}+(C+1)^{2} 2^{\frac{k+1}{2}} n^{\frac{k-1}{k}}\left((C+1) \sqrt{\frac{n \Delta}{b}}\right)^{\frac{1}{k}},
\end{aligned}
$$

where the first step uses (33) and Lemma 5.3; the second step applies the inductive hypothesis; and the last step can be verified in a straightforward manner by examining the cases $\Delta \leqslant n / b$ and $\Delta \geqslant n / b$. Setting

$$
b=(C+1)^{2} 2^{k}\left(\frac{n}{\Delta}\right)^{1-\frac{2}{k+1}}
$$

in (43) now yields (41), completing the inductive step. Note that our choice of parameter meets the requirement $b \geqslant 1$, as one can see from (42).

\section{$6 \quad k$-ELEMENT DISTINCTNESS}

For an integer $k$, recall that the threshold function $\mathrm{THR}_{k}:\{0,1\}^{*} \rightarrow$ $\{0,1\}$ is given by

$$
\operatorname{THR}_{k}(x)= \begin{cases}1 & \text { if }|x| \geqslant k, \\ 0 & \text { otherwise. }\end{cases}
$$

As a generate case, we have

$$
\mathrm{THR}_{0} \equiv 1 .
$$

In the $k$-element distinctness problem, the input is a list of $n$ integers from some range of size $r$, and the objective is to determine whether some integer occurs at least $k$ times. Traditionally, the input to $k$-element distinctness is represented by a Boolean matrix $x \in$ $\{0,1\}^{n \times r}$ with precisely one nonzero entry in each row. We depart from tradition by allowing the input $x \in\{0,1\}^{n \times r}$ to be an arbitrary matrix with at most $n$ ones. Formally, we define the $k$-element distinctness function $\mathrm{ED}_{n, r, k}:\{0,1\}_{\leqslant n}^{n r} \rightarrow\{0,1\}$ by

$$
\mathrm{ED}_{n, r, k}(x)=\neg \bigvee_{i=1}^{r} \operatorname{THR}_{k}\left(x_{1, i} x_{2, i} \ldots x_{n, i}\right) .
$$

Since our focus is on upper bounds, working with the more general domain makes our results stronger. Our main result in this section is as follows.

THEOREM 6.1. Let $k \geqslant 1$ be a fixed integer. Then for all integers $n, r \geqslant 1$ and all reals $0<\epsilon \leqslant 1 / 2$,

$$
\begin{aligned}
& \operatorname{deg}_{\epsilon}\left(\operatorname{ED}_{n, r, k}\right)=O\left(\sqrt{n} \min \{n, r\}^{\frac{1}{2}-\frac{1}{4\left(1-2^{-k}\right)}}\left(\log \frac{1}{\epsilon}\right)^{\frac{1}{4\left(1-2^{-k}\right)}}\right) \\
& +O\left(\sqrt{n \log \frac{1}{\epsilon}}\right) \text {, }
\end{aligned}
$$

where the approximating polynomial is given explicitly in each case. 
Taking $\epsilon=1 / 3$ in this result settles Theorem 1.1 from the introduction. To prove Theorem 6.1, we will need to consider a more general class of functions. For nonnegative integers $n, r, k$ and a real number $\Delta \geqslant 1$, we define

$$
D(n, r, k, \Delta)=\max _{F} \operatorname{deg}_{2^{-\Delta}}(F),
$$

where the maximum is over all functions $F:\{0,1\}_{\leqslant n}^{N} \rightarrow\{0,1\}$ for some $N$ that are expressible as

$$
F(x)=\bigvee_{i=1}^{r} \operatorname{THR}_{k_{i}}\left(\left.x\right|_{S_{i}}\right)
$$

for some pairwise disjoint sets $S_{1}, S_{2}, \ldots, S_{r} \subseteq\{1,2, \ldots, N\}$ and some $k_{1}, k_{2}, \ldots, k_{r} \in\{0,1,2, \ldots, k\}$. The four-argument quantity $D$ that we have just defined is unrelated to the three-argument quantity $D$ from Section 5 . We abbreviate

$$
D(n, \infty, k, \Delta)=\max _{r \geqslant 1} D(n, r, k, \Delta) .
$$

By definition,

$$
\operatorname{deg}_{\epsilon}\left(\mathrm{ED}_{n, r, k}\right) \leqslant D\left(n, r, k, \log \frac{1}{\epsilon}\right), \quad 0<\epsilon \leqslant \frac{1}{2} .
$$

Our analysis of $D(n, r, k, \Delta)$ proceeds by induction on $k$. As the base cases, we have

$$
D(n, \infty, 0, \Delta)=0
$$

by (44), and

$$
D(n, \infty, 1, \Delta)=C \sqrt{n \Delta}
$$

by Theorem 4.6 for some constant $C \geqslant 1$. Also, Fact 2.1 implies that

$$
D(n, \infty, k, \Delta) \leqslant n .
$$

\subsection{A Recursive Bound for Small Range}

To implement the inductive step, we derive two complementary recursive bounds for $D(n, r, k, \Delta)$. The first of these bounds, presented below, is tailored to the case when $n \geqslant k r$.

LEMMA 6.2. There is a constant $C \geqslant 1$ such that for all positive integers $n, r, k$ and all reals $\Delta \geqslant 1$,

$$
D(n, r, k, \Delta) \leqslant C \cdot \sqrt{1+\frac{n}{k r}} \cdot(D(2 k r, r, k, \Delta+1)+\Delta) .
$$

Proof. Since $D$ is monotonically increasing in every argument, the lemma holds trivially for $n<k r$. In what follows, we consider the complementary case

$$
n \geqslant k r \text {. }
$$

Consider an arbitrary function $F:\{0,1\}_{\leqslant n}^{N} \rightarrow\{0,1\}$ of the form

$$
F(x)=\bigvee_{i=1}^{r} \operatorname{THR}_{k_{i}}\left(\left.x\right|_{S_{i}}\right)
$$

for some pairwise disjoint sets $S_{1}, S_{2}, \ldots, S_{r} \subseteq\{1,2, \ldots, N\}$ and $k_{1}, k_{2}, \ldots, k_{r} \in\{0,1,2, \ldots, k\}$. By discarding any irrelevant variables among $x_{1}, x_{2}, \ldots, x_{N}$, we may assume that $S_{1} \cup S_{2} \cup \cdots \cup S_{r}=$ $\{1,2, \ldots, N\}$. Then by the pigeonhole principle, any input $x$ with Hamming weight at least $k r$ satisfies at least one of the disjuncts in (50). Therefore,

$$
F(x)=1, \quad x \in\{0,1\}_{\geqslant k r}^{N} .
$$

For $i \geqslant k r$, define $F_{i}:\{0,1\}_{\leqslant i}^{N} \rightarrow\{0,1\}$ by

$$
F_{i}(x)= \begin{cases}F(x) & \text { if }|x| \leqslant k r \\ 1 & \text { otherwise }\end{cases}
$$

Then

$$
\begin{aligned}
\operatorname{deg}_{2^{-\Delta}}(F) & =\operatorname{deg}_{2^{-\Delta}}\left(F_{n}\right) \\
& =\operatorname{deg}_{2^{-\Delta}}\left(1-F_{n}\right) \\
& \leqslant c \sqrt{\frac{n}{k r}} \cdot\left(\operatorname{deg}_{2^{-\Delta-1}}\left(1-F_{2 k r}\right)+\Delta+1\right) \\
& =c \sqrt{\frac{n}{k r}} \cdot\left(\operatorname{deg}_{2^{-\Delta-1}}\left(F_{2 k r}\right)+\Delta+1\right) \\
& \leqslant c \sqrt{\frac{n}{k r}} \cdot(D(2 k r, r, k, \Delta+1)+\Delta+1)
\end{aligned}
$$

for some absolute constant $c \geqslant 1$ and all $\Delta \geqslant 1$, where the first and last steps use (51), and the third step applies (49) and the extension theorem (Theorem 3.1) with $m=k r$ and $\epsilon=\delta=2^{-\Delta-1}$. As a result, the lemma holds with $C=2 c$.

\subsection{A Recursive Bound for Large Range}

We now derive an alternate upper bound on $D(n, r, k, \Delta)$, with no dependence on the range parameter $r$. This result addresses the case of large $r$ and complements Lemma 6.2.

LEMMA 6.3. There is a constant $C \geqslant 1$ such that for all integers $n, k \geqslant 1$ and all reals $\Delta, b \geqslant 1$,

$$
\begin{aligned}
& D(n, \infty, k, \Delta) \leqslant C \sqrt{n b \Delta}+C\left(1+\frac{1}{\sqrt{k}}\left(\frac{n}{b \Delta}\right)^{1 / 4}\right) \times \\
& \quad \times\left(D\left(\lfloor C k \sqrt{n b \Delta}\rfloor, \infty, k-1, C \sqrt{\frac{n \Delta}{b}}+1\right)+\sqrt{\frac{n \Delta}{b}}\right) .
\end{aligned}
$$

Proof. Consider an arbitrary function $F:\{0,1\}_{\leqslant n}^{N} \rightarrow\{0,1\}$ of the form

$$
F(x)=\bigvee_{i=1}^{r} \operatorname{THR}_{k_{i}}\left(\left.x\right|_{S_{i}}\right)
$$

for some integer $r \geqslant 1$, some pairwise disjoint sets $S_{1}, S_{2}, \ldots, S_{r} \subseteq$ $\{1,2, \ldots, N\}$, and some $k_{1}, k_{2}, \ldots, k_{r} \in\{0,1,2, \ldots, k\}$. If $k_{i}=0$ for some $i$, then the corresponding term in (53) is the constant function 1, resulting in $\operatorname{deg}_{0}(F)=0$. In what follows, we treat the complementary case when $k_{i} \geqslant 1$ for each $i$.

Rewriting (53),

$$
F(x)=\bigvee_{i=1}^{r} \bigvee_{j \in S_{i}} x_{j} \wedge \operatorname{THR}_{k_{i}-1}\left(\left.x\right|_{S_{i} \backslash\{j\}}\right)
$$

As this representation suggests, our intention is to bound the approximate degree of $F$ by appeal to Theorem 5.2. 
CLAIM 6.4. Fix a subset $S_{i}^{\prime} \subseteq S_{i}$ for each $i=1,2, \ldots, r$. Then for $\Delta \geqslant 1$,

$$
\begin{aligned}
\operatorname{deg}_{2^{-}}\left(\bigvee_{i=1}^{r} \bigvee_{j \in S_{i}^{\prime}} \operatorname{THR}_{k_{i}-1}\left(\left.x\right|_{S_{i} \backslash\{j\}}\right)\right) \\
\quad \leqslant D\left(n, \sum_{i=1}^{r}\left|S_{i}^{\prime}\right|, k-1, \Delta\right)+\sum_{i=1}^{r}\left|S_{i}^{\prime}\right| .
\end{aligned}
$$

We will settle Claim 6.4 once we complete the main proof. In light of this claim, the representation (54) shows that $F(x)=\bigvee_{i=1}^{N} x_{i} \wedge f_{i}(x)$ for some functions $f_{i}$ such that

$$
\operatorname{deg}_{2^{-}}\left(\bigvee_{i \in S} f_{i}\right) \leqslant D(n,|S|, k-1, \Delta)+|S|
$$

for all $S \subseteq\{1,2, \ldots, N\}$ and all $\Delta \geqslant 1$. Then for some absolute constants $c^{\prime}, c^{\prime \prime} \geqslant 1$ and all $\Delta \geqslant 1$ and $b \geqslant 1$, we have

$$
\begin{array}{r}
\operatorname{deg}_{2^{-\Delta}}(F) \leqslant c^{\prime} \sqrt{n b \Delta}+\max _{\substack{S \subseteq\{1, \ldots, N\} \\
|S| \leqslant c^{\prime} \sqrt{n b \Delta}}} \operatorname{deg}_{2^{-\Delta} \exp \left(-c^{\prime} \sqrt{n \Delta / b}\right)}\left(\bigvee_{i \in S} f_{i}\right) \\
\leqslant 2 c^{\prime} \sqrt{n b \Delta}+D\left(n,\left\lceil c^{\prime} \sqrt{n b \Delta}\right\rceil, k-1, \Delta+\frac{c^{\prime}}{\ln 2} \sqrt{\frac{n \Delta}{b}}\right) \\
\leqslant 2 c^{\prime} \sqrt{n b \Delta}+c^{\prime \prime} \cdot \sqrt{1+\frac{n}{k \cdot c^{\prime} \sqrt{n b \Delta}}} \times \\
\quad \times\left(D\left(2 k\left\lceil c^{\prime} \sqrt{n b \Delta}\right\rceil, \infty, k-1, \Delta+\frac{c^{\prime}}{\ln 2} \sqrt{\frac{n \Delta}{b}}+1\right)\right. \\
\left.+\Delta+\frac{c^{\prime}}{\ln 2} \sqrt{\frac{n \Delta}{b}}\right),
\end{array}
$$

where the first step applies Theorem 5.2, the second step uses (55), and the final step follows from (46) for $k=1$ and from Lemma 6.2 for $k \geqslant 2$. This directly implies (52) for $\Delta \leqslant n / b$. In the complementary case $\Delta>n / b$, the right-hand side of (52) exceeds $n$ and therefore the bound follows trivially from (48).

Proof of Claim 6.4. To start with,

$$
\begin{aligned}
\bigvee_{i=1}^{r} \bigvee_{j \in S_{i}^{\prime}} \operatorname{THR}_{k_{i}-1}\left(\left.x\right|_{S_{i} \backslash\{j\}}\right) & \\
= & \bigvee_{i: S_{i}^{\prime} \neq \varnothing} \bigvee_{j \in S_{i}^{\prime}} \operatorname{THR}_{k_{i}-1}\left(\left.x\right|_{S_{i} \backslash\{j\}}\right) \\
& =\bigvee_{i: S_{i}^{\prime} \neq \varnothing} \operatorname{THR}_{k_{i}-1-\min }\left\{|x|_{S_{i}^{\prime}}|,| S_{i}^{\prime} \mid-1\right\}
\end{aligned}
$$

Considering the possible values for the Hamming weight of each $\left.x\right|_{S_{i}^{\prime}}$, we arrive at the representation

$$
\begin{aligned}
& \bigvee_{i=1}^{r} \bigvee_{j \in S_{i}^{\prime}} \mathrm{THR}_{k_{i}-1}\left(\left.x\right|_{S_{i} \backslash\{j\}}\right)=\sum_{\ell_{1}=0}^{\left|S_{1}^{\prime}\right|} \cdots \sum_{\ell_{r}=0}^{\left|S_{r}^{\prime}\right|} \mathrm{I}\left[|x|_{S_{i}^{\prime}} \mid=\ell_{i} \quad \forall i\right] \\
& \quad \times\left(\bigvee_{i: S_{i}^{\prime} \neq \varnothing} \operatorname{THR}_{k_{i}-1-\min }\left\{\ell_{i},\left|S_{i}^{\prime}\right|-1\right\}\right. \\
& \left.\left(\left.x\right|_{S_{i} \backslash S_{i}^{\prime}}\right)\right)
\end{aligned}
$$

The indicator functions in this summation are mutually exclusive in that for any given value of $x$, precisely one of them is nonzero. As a result, the right-hand side of (56) can be approximated pointwise to within $2^{-\Delta}$ by replacing each parenthesized expression with its $2^{-\Delta}$-error approximant, which by definition can be chosen to have degree at most $D\left(n, \sum\left|S_{i}^{\prime}\right|, k-1, \Delta\right)$. This completes the proof since each indicator function in (56) depends on only $\sum\left|S_{i}^{\prime}\right|$ Boolean variables and is therefore a polynomial of degree at most $\sum\left|S_{i}^{\prime}\right|$.

\subsection{Solving the Recurrence}

It remains to solve the newly obtained recurrences. We first solve the recurrence given by (47) and Lemma 6.3, corresponding to the infinite-range case.

THEOREM 6.5 (RANGE-INDEPENDENT BOUND). There is a constant $c \geqslant 1$ such that for all positive integers $n$ and $k$, and all reals $\Delta \geqslant 1$,

$$
D(n, \infty, k, \Delta) \leqslant c^{k} \sqrt{k !} \cdot n^{1-\frac{1}{4\left(1-2^{-k}\right)}} \Delta^{\frac{1}{4\left(1-2^{-k}\right)}} .
$$

Proof. We will prove (57) for $c=(4 C)^{2}$, where $C \geqslant 1$ is the larger of the constants in (47) and Lemma 6.3. The proof is by induction on $k$. The base case $k=1$ is immediate from (47). For the inductive step, let $k \geqslant 2$ be arbitrary. When $\Delta>n$, the righthand side of (57) exceeds $n$ and therefore the bound is immediate from (48). In what follows, we assume that

$$
1 \leqslant \Delta \leqslant n
$$

Let $b \geqslant 1$ be a parameter to be fixed later. By Lemma 6.3,

$$
\begin{aligned}
D(n, \infty, k, \Delta) \leqslant C & \sqrt{n b \Delta}+C\left(1+\frac{1}{\sqrt{k}}\left(\frac{n}{b \Delta}\right)^{\frac{1}{4}}\right)\left(\sqrt{\frac{n \Delta}{b}}\right. \\
& \left.+D\left(\lfloor C k \sqrt{n b \Delta}\rfloor, \infty, k-1, C \sqrt{\frac{n \Delta}{b}}+1\right)\right) .
\end{aligned}
$$

It follows that

$$
\begin{aligned}
D(n, \infty, k, \Delta) \leqslant C \sqrt{k n b \Delta} & +2 C\left(\frac{n}{k b \Delta}\right)^{\frac{1}{4}}\left(\sqrt{\frac{n \Delta}{b}}\right. \\
& \left.+D\left(\lfloor C k \sqrt{n b \Delta}\rfloor, \infty, k-1, C \sqrt{\frac{n \Delta}{b}}+1\right)\right),
\end{aligned}
$$

as one can verify from the previous step if $n \geqslant k b \Delta$ and from (48) if $n<k b \Delta$. Applying the inductive hypothesis,

$$
\begin{aligned}
& D(n, \infty, k, \Delta) \leqslant C \sqrt{k n b \Delta}+2 C\left(\frac{n}{k b \Delta}\right)^{\frac{1}{4}}\left(\sqrt{\frac{n \Delta}{b}}\right. \\
& \left.+c^{k-1} \sqrt{(k-1) !} \cdot(C k \sqrt{n b \Delta})^{1-\frac{1}{4\left(1-2^{-k+1}\right)}}\left(C \sqrt{\frac{n \Delta}{b}}+1\right)^{\frac{1}{4\left(1-2^{-k+1}\right)}}\right) .
\end{aligned}
$$

Now the bound

$$
\begin{aligned}
& D(n, \infty, k, \Delta) \leqslant C \sqrt{k n b \Delta}+2 C\left(\frac{n}{k b \Delta}\right)^{\frac{1}{4}} \times \\
& \times 4 c^{k-1} \sqrt{(k-1) !} \cdot(C k \sqrt{n b \Delta})^{1-\frac{1}{4\left(1-2^{-k+1}\right)}}\left(C \sqrt{\frac{n \Delta}{b}}\right)^{\frac{1}{4\left(1-2^{-k+1}\right)}}
\end{aligned}
$$


is immediate from the previous step if $n \geqslant b / \Delta$ and from (48) if $n<b / \Delta$. Rearranging, we find that

$$
\begin{aligned}
& D(n, \infty, k, \Delta) \\
\leqslant & C \sqrt{k n b \Delta}\left(1+C\left(\frac{n}{\Delta}\right)^{\frac{1}{4}} \cdot 8 c^{k-1} \sqrt{(k-1) !} b^{-\frac{1}{4}-\frac{1}{4\left(1-2^{-k+1}\right)}}\right) .
\end{aligned}
$$

The right-hand side is minimized at

$$
b=\left(C\left(\frac{n}{\Delta}\right)^{\frac{1}{4}} \cdot 8 c^{k-1} \sqrt{(k-1) !}\right)^{\frac{2^{k+1}-4}{2^{k}-1}},
$$

which in view of $(58)$ is a real number in $[1, \infty)$ and therefore a legitimate parameter setting. Making this substitution in (59), we arrive at

$$
\begin{aligned}
& D(n, \infty, k, \Delta) \leqslant 2 C \sqrt{k n \Delta}\left(C\left(\frac{n}{\Delta}\right)^{\frac{1}{4}} \cdot 8 c^{k-1} \sqrt{(k-1) !}\right)^{\frac{2^{k}-2}{2^{k-1}}} \\
& \leqslant 2 C^{2} \cdot 8 c^{k-1} \sqrt{k ! n \Delta\left(\frac{n}{\Delta}\right)^{\frac{2^{k-1}-1}{2^{k}-1}}} \\
& =c^{k} \sqrt{k !} n^{1-\frac{1}{4\left(1-2^{-k}\right)}} \Delta^{\frac{1}{4\left(1-2^{-k}\right)}} \text {. }
\end{aligned}
$$

This completes the inductive step and settles (57).

By combining the previous result with an application of Lemma 6.2, we will now prove our main bound on $D(n, r, k, \Delta)$.

Theorem 6.6 (RANGe-DEPENDENT Bound). There is a constant $c \geqslant 1$ such that for all positive integers $n, r, k$ and all reals $\Delta \geqslant 1$,

$$
D(n, r, k, \Delta) \leqslant c^{k} \sqrt{k !}\left(\sqrt{n} \min \{n, k r\}^{\frac{1}{2}-\frac{1}{4\left(1-2^{-k}\right)}} \Delta^{\frac{1}{4\left(1-2^{-k}\right)}}+\sqrt{n \Delta}\right) .
$$

Proof. The bound follows from Theorem 6.5 if $k r \geqslant n$; and from (48) if $\Delta \geqslant n$. As a result, we may assume that

$$
\begin{aligned}
& n>k r, \\
& n>\Delta .
\end{aligned}
$$

In what follows, let $C \geqslant 1$ denote the larger of the constants in Lemma 6.2 and Theorem 6.5. Then

$$
\begin{aligned}
& D(n, r, k, \Delta) \\
& \leqslant D\left(n, r+\left\lceil\frac{\Delta}{k}\right\rceil, k, \Delta\right) \\
& \leqslant C \cdot \sqrt{1+\frac{n}{k r+k\lceil\Delta / k\rceil} \cdot\left(D\left(2 k r+2 k\left\lceil\frac{\Delta}{k}\right\rceil, \infty, k, \Delta+1\right)+\Delta\right)} \\
& \leqslant 2 C \cdot \sqrt{\frac{n}{k r+k\lceil\Delta / k\rceil}} \cdot\left(D\left(2 k r+2 k\left\lceil\frac{\Delta}{k}\right\rceil, \infty, k, \Delta+1\right)+\Delta\right) \\
& \leqslant 2 C \cdot \sqrt{\frac{n}{k r+k\lceil\Delta / k\rceil}} \\
& \quad \times\left(C^{k \sqrt{k !}}\left(2 k r+2 k\left\lceil\frac{\Delta}{k}\right\rceil\right)^{1-\frac{1}{4\left(1-2^{-k}\right)}}(\Delta+1)^{\frac{1}{4\left(1-2^{-k}\right)}}+\Delta\right)
\end{aligned}
$$

$$
\begin{aligned}
& \leqslant 2 C \cdot \sqrt{\frac{n}{k r+k\lceil\Delta / k\rceil}} \\
& \times 2 C^{k} \sqrt{k !}\left(2 k r+2 k\left\lceil\frac{\Delta}{k}\right\rceil\right)^{1-\frac{1}{4\left(1-2^{-k}\right)}}(\Delta+1)^{\frac{1}{4\left(1-2^{-k}\right)}} \\
& =4 C^{k+1} \sqrt{k !} \cdot \sqrt{2 n}\left(2 k r+2 k\left\lceil\frac{\Delta}{k}\right\rceil\right)^{\frac{1}{2}-\frac{1}{4\left(1-2^{-k}\right)}}(\Delta+1)^{\frac{1}{4\left(1-2^{-k}\right)}},
\end{aligned}
$$

where the first step is valid because $D$ is monotonically increasing in every argument; the second step applies Lemma 6.2; the third step uses (60) and (61); and the fourth step applies Theorem 6.5. This completes the proof of the theorem for $n>k r$.

Equation (45) and Theorem 6.6 establish the main result of this section, Theorem 6.1. We note that with a more careful analysis, the multiplicative factor $\sqrt{k !}$ in Theorems 6.5 and 6.6 can be improved to a slightly smaller quantity, still of the order of $k^{O(k)}$.

\section{SURJECTIVITY}

For positive integers $n$ and $r$, the surjectivity problem is to determine whether a given mapping $\{1,2, \ldots, n\} \rightarrow\{1,2, \ldots, r\}$ is surjective. Traditionally, the input to this problem is represented by a Boolean matrix $x \in\{0,1\}^{n \times r}$ with precisely one nonzero entry in every row. Analogous to our work on element distinctness in the previous section, we depart from tradition by allowing arbitrary matrices $x \in$ $\{0,1\}^{n \times r}$ with at most $n$ ones. Specifically, we define the surjectivity ${\text { function } \mathrm{SURJ}_{n, r}:\{0,1\}^{n r} \rightarrow n}_{\rightarrow}^{n r}\{0,1\}$ by

$$
\operatorname{SURJ}_{n, r}(x)=\bigwedge_{j=1}^{r} \bigvee_{i=1}^{n} x_{i, j}
$$

This formalism corresponds to determining the surjectivity of arbitrary relations on $\{1,2, \ldots, n\} \times\{1,2, \ldots, r\}$, including functions $\{1,2, \ldots, n\} \rightarrow\{1,2, \ldots, r\}$ as a special case. Since we are interested in upper bounds, working in this more general setting makes our results stronger.

In this extended abstract, we bound the approximate degree of surjectivity with the error parameter set to $\epsilon=1 / 3$. This setting covers most applications of interest and allows for a shorter and simpler proof. The case of arbitrary $\epsilon$ is treated in the full version of this paper.

Theorem (Restatement of Theorem 1.3). For all positive integers $n$ and $r$,

$$
\begin{aligned}
& \operatorname{deg}_{1 / 3}\left(\operatorname{SURJ}_{n, r}\right)=O\left(\sqrt{n} \cdot r^{1 / 4}\right) \quad(r \leqslant n), \\
& \operatorname{deg}_{1 / 3}\left(\operatorname{SURJ}_{n, r}\right)=0 \quad(r>n),
\end{aligned}
$$

where the approximating polynomial is given explicitly in each case.

The theorem shows that $\operatorname{deg}_{1 / 3}\left(\operatorname{SURJ}_{n, r}\right)=O\left(n^{3 / 4}\right)$ for all integers $r$, disproving the conjecture of Bun and Thaler [14] that the $1 / 3$ approximate degree of $\operatorname{SURJ}_{n, \Omega(n)}$ is linear in $n$.

Proof. The identity SURJ $n, r \equiv 0$ for $r>n$ implies (63) directly. The proof of (62) involves two steps. First, we construct an explicit real-valued function $\widetilde{\mathrm{SURJ}}_{n, r}$ that approximates $\mathrm{SURJ}_{n, r}$ pointwise and is representable by a linear combination of conjunctions with reasonably small coefficients. Then, we replace each conjunction 
in this linear combination by an approximating polynomial of low degree.

In more detail, let $m \geqslant 1$ be an integer parameter to be chosen later. Recall from (6) and Proposition 2.3 that the Chebyshev polynomial $T_{m}$ obeys

$$
\begin{array}{ll}
\left|T_{m}(t)\right| \leqslant 1, & -1 \leqslant t \leqslant t, \\
T_{m}\left(1+\frac{1}{r}\right) \geqslant 1+\frac{m^{2}}{r} . &
\end{array}
$$

As a result, $\mathrm{SURJ}_{n, r}$ is approximated pointwise within $1 /\left(1+\frac{m^{2}}{r}\right)$ by

$$
\widetilde{\operatorname{SURJ}}_{n, r}(x)=\frac{1}{T_{m}\left(1+\frac{1}{r}\right)} \cdot T_{m}\left(\frac{1}{r}+\frac{1}{r} \sum_{j=1}^{r} \bigvee_{i=1}^{n} x_{i, j}\right) \text {. }
$$

Therefore,

$$
E\left(\mathrm{SURJ}_{n, r}, d\right) \leqslant \frac{1}{1+\frac{m^{2}}{r}}+E\left(\widetilde{\mathrm{SURJ}}_{n, r}, d\right), \quad d=1,2,3, \ldots
$$

To estimate the rightmost term in (64), use the factored representation (8) to write

$$
\begin{aligned}
& \widetilde{\operatorname{SURJ}}_{n, r}(x) \\
& =\frac{2^{m-1}}{T_{m}\left(1+\frac{1}{r}\right)} \cdot \prod_{i=1}^{m}\left(\frac{1}{r}+\frac{1}{r} \sum_{j=1}^{r}\left(\bigvee_{i=1}^{n} x_{i, j}\right)-\cos \frac{(2 i-1) \pi}{2 m}\right) \\
& =\frac{2^{m-1}}{T_{m}\left(1+\frac{1}{r}\right)} \cdot \prod_{i=1}^{m}\left(\frac{1}{r}+1-\frac{1}{r} \sum_{j=1}^{r} \prod_{i=1}^{n} \overline{x_{i, j}}-\cos \frac{(2 i-1) \pi}{2 m}\right) .
\end{aligned}
$$

Multiplying out shows that $\widetilde{\operatorname{SURJ}}_{n, r}(x)$ is a linear combination of conjunctions with real coefficients whose absolute values sum to ${ }_{2} \mathrm{O}(\mathrm{m})$. By Corollary 4.7, each of these conjunctions can be approximated by a polynomial of degree $d$ to within $2^{-\Theta\left(d^{2} / n\right)}$ pointwise. We conclude that

$$
E\left(\widetilde{\operatorname{SURJ}}_{n, r}, d\right) \leqslant 2^{O(m)} \cdot 2^{-\Theta\left(d^{2} / n\right)},
$$

which along with (64) gives

$$
E\left(\operatorname{SURJ}_{n, r}, d\right) \leqslant \frac{1}{1+\frac{m^{2}}{r}}+2^{O(m)} \cdot 2^{-\Theta\left(d^{2} / n\right)} .
$$

Now (62) follows by taking $m=\lceil\sqrt{3 r}\rceil$ and $d=\Theta\left(\sqrt{n} \cdot r^{1 / 4}\right)$. The approximating polynomial in question is given explicitly because every stage of our proof, including the appeal to Corollary 4.7, is constructive.

\section{ACKNOWLEDGMENTS}

The author was supported in part by NSF CAREER award CCF1149018 and an Alfred P. Sloan Foundation Research Fellowship. The author is thankful to Paul Beame, Aleksandrs Belovs, Mark Bun, Robin Kothari, Justin Thaler, Emanuele Viola, and Ronald de Wolf for valuable comments on an earlier version of this paper. The author is further indebted to Mark, Robin, and Justin for stimulating discussions and for sharing a preliminary version of their manuscript [13], which inspired the title of this paper.

\section{REFERENCES}

[1] Scott Aaronson, Shalev Ben-David, and Robin Kothari. 2016. Separations in query complexity using cheat sheets. In STOC. 863-876.

[2] Scott Aaronson and Yaoyun Shi. 2004. Quantum lower bounds for the collision and the element distinctness problems. 7. ACM 51, 4 (2004), 595-605.

[3] Andris Ambainis. 2005. Polynomial Degree and Lower Bounds in Quantum Complexity: Collision and Element Distinctness with Small Range. Theory of Computing 1, 1 (2005), 37-46.

[4] Andris Ambainis. 2006. Polynomial degree vs. quantum query complexity. 7 . Comput. Syst. Sci. 72, 2 (2006), 220-238.

[5] Andris Ambainis. 2007. Quantum Walk Algorithm for Element Distinctness. SIAM 7. Comput. 37, 1 (2007), 210-239.

[6] Andris Ambainis, Andrew M. Childs, Ben Reichardt, Robert Špalek, and Shengyu Zhang. 2010. Any AND-OR Formula of Size $N$ can be Evaluated in time $N^{1 / 2+o(1)}$ on a Quantum Computer. SIAM 7. Comput. 39, 6 (2010), 2513-2530.

[7] Robert Beals, Harry Buhrman, Richard Cleve, Michele Mosca, and R. de Wolf. 2001. Quantum lower bounds by polynomials. F. ACM 48, 4 (2001), 778-797.

[8] Paul Beame and Widad Machmouchi. 2012. The quantum query complexity of $\mathrm{AC}^{0}$. Quantum Information \& Computation 12, 7-8 (2012), 670-676.

[9] Aleksandrs Belovs. 2012. Learning-Graph-Based Quantum Algorithm for $k$ Distinctness. In FOCS. 207-216.

[10] Aleksandrs Belovs and Robert Špalek. 2013. Adversary lower bound for the $k$-sum problem. In Innovations in Theoretical Computer Science (ITCS). 323-328.

[11] Harry Buhrman, Richard Cleve, R. de Wolf, and Christof Zalka. 1999. Bounds for Small-Error and Zero-Error Quantum Algorithms. In FOCS. 358-368.

[12] Harry Buhrman, Christoph Dürr, Mark Heiligman, Peter Høyer, Frédéric Magniez, Miklos Santha, and Ronald de Wolf. 2005. Quantum Algorithms for Element Distinctness. SIAM f. Comput. 34, 6 (2005), 1324-1330.

[13] Mark Bun, Robin Kothari, and Justin Thaler. 2017. The Polynomial Method Strikes Back: Tight Quantum Query Bounds Via Dual Polynomials. ECCC Report TR17-169. (2017)

[14] Mark Bun and Justin Thaler. 2017. A Nearly Optimal Lower Bound on the Approximate Degree of $\mathrm{AC}^{0}$. In FOCS. 1-12.

[15] Andrew M. Childs and Jason M. Eisenberg. 2005. Quantum algorithms for subset finding. Quantum Information \& Computation 5, 7 (2005), 593-604.

[16] Andrew Drucker and Ronald de Wolf. 2011. Quantum Proofs for Classical Theorems. Theory of Computing, Graduate Surveys 2 (2011), 1-54

[17] Andrew Drucker and Ronald de Wolf. 2011. Uniform approximation by (quantum) polynomials. Quantum Information \& Computation 11, 3\&4 (2011), 215-225.

[18] Edward Farhi, Jeffrey Goldstone, and Sam Gutmann. 2008. A Quantum Algorithm for the Hamiltonian NAND Tree. Theory of Computing 4, 1 (2008), 169-190.

[19] Jeff Kahn, Nathan Linial, and Alex Samorodnitsky. 1996. Inclusion-Exclusion: Exact and Approximate. Combinatorica 16, 4 (1996), 465-477.

[20] Samuel Kutin. 2005. Quantum Lower Bound for the Collision Problem with Small Range. Theory of Computing 1, 1 (2005), 29-36.

[21] Urmila Mahadev and Ronald de Wolf. 2015. Rational approximations and quantum algorithms with postselection. Quantum Information \& Computation 15, 3\&4 (2015), 295-307.

[22] Noam Nisan and Mario Szegedy. 1994. On the degree of Boolean functions as real polynomials. Computational Complexity 4 (1994), 301-313.

[23] Ryan O'Donnell and Rocco A. Servedio. 2010. New degree bounds for polynomial threshold functions. Combinatorica 30, 3 (2010), 327-358.

[24] Ramamohan Paturi. 1992. On the degree of polynomials that approximate symmetric Boolean functions. In STOC. 468-474.

[25] Alexander A. Sherstov. 2009. Approximate Inclusion-Exclusion for Arbitrary Symmetric Functions. Computational Complexity 18, 2 (2009), 219-247. Preliminary version in CCC, 2008.

[26] Alexander A. Sherstov. 2013. Making polynomials robust to noise. Theory of Computing 9 (2013), 593-615. Preliminary version in STOC, 2012

[27] Alexander A. Sherstov. 2018. Algorithmic polynomials. In Electronic Colloquium on Computational Complexity (ECCC). Report TR18-010.

[28] Ronald de Wolf. 2008. A note on quantum algorithms and the minimal degree of $\epsilon$-error polynomials for symmetric functions. Quantum Information and Computation 8, 10 (2008), 943-950. 